Modelling Simul. Mater. Sci. Eng., in press

\title{
Length scale effects and multiscale modeling of thermally induced phase transformation kinetics in NiTi SMA
}

\author{
George N. Frantziskonis ${ }^{1,2, *}$ and Sourav Gur ${ }^{1}$ \\ ${ }^{1}$ Civil Engineering and Engineering Mechanics, University of Arizona, Tucson, AZ 85721 USA \\ ${ }^{2}$ Materials Science and Engineering, University of Arizona, Tucson, AZ 85721 USA \\ *Corresponding author Email: frantzis@email.arizona.edu (G. N. Frantziskonis)
}

\begin{abstract}
Thermally induced phase transformation in NiTi shape memory alloys (SMA) shows strong size and shape, collectively termed length scale effects, at the nano to micrometer scales, and that has important implications for the design and use of devices and structures at such scales. This paper, based on a recently developed multiscale model that utilizes molecular dynamics (MD) simulations at small scales and MD-verified phase field (PhF) simulations at larger scales, reports results on specific length scale effects, i.e. length scale effects in martensite phase fraction evolution, transformation temperatures (martensite and austenite start and finish) and in the thermally cyclic transformation between austenitic and martensitic phase. The multiscale study identifies saturation points for length scale effects and studies, for the first time, the length scale effect on the kinetics (i.e. developed internal strains) in the B19 phase during phase transformation. The major part of the work addresses small scale single crystals in specific orientations. However, the multiscale method is used in a unique and novel way to indirectly study length scale and grain size effects on evolution kinetics in polycrystalline $\mathrm{NiTi}$, and to compare the simulation results to experiments. The interplay of the grain size and the length scale effect on the thermally induced martensite phase fraction (MPF) evolution is also shown in this present study. Finally, the multiscale coupling results are employed to improve phenomenological material models for NiTi SMA.
\end{abstract}

Keywords: NiTi SMA, length scale effect, single crystal, polycrystal, multiscale coupling and material model.

\section{Introduction}

Experimental and/or computational study of the martensitic phase transformation in SMAs such as NiTi is demanding, mainly because of the complexity and stochasticity in the formation of different martensite variants [1-8]. Free surfaces interfere with the formation of variants, leading, especially at small scales where the ratio of free surface area to volume is large, to significant size and scale effects, collectively termed as length scale effects [9-15]. Not only the length scales affect the phase fraction evolution and the thermally cyclic hysteresis, but also affect the transformation temperatures (martensite and austenite start and finish) and the overall constitutive response of the material. Size effects have been studied recently [8,11-15], but shape effects have not, even though a lot would be learned from such studies that would aid the design and development of nanoscale SMA-based devices such as sensors and actuators. Computational studies employing classical MD simulations at the atomic level [11,13-15] or stochastic phase field simulation methods at the mesoscale level 
$[8,16]$ demonstrate size effects (due to the presence of free surfaces) on the phase transformation process of NiTi SMA. Findings (experimental or computational) include: (a) equiatomic NiTi thin samples [9,10,17] and nanoparticles [11,13] showing nucleation and growth of unexpected stable crystal structures at the nanometer scale; (b) multiple transformation paths from B2 to B19 $\square$ variants $[13,18,19]$ in nanograins NiTi; and (c) highly stable square nanowires against torsional buckling, as compared to circular ones [20].

During thermally induced martensitic phase transformation internal strains develop in both B2 (austenite) and B19 (martensite) phases. Recent experimental studies by Ahadi and Sun [21-23] demonstrate the effect on grain size on the stress-induced martensitic phase transition process, in polycrystalline superelastic NiTi SMA, at the nanoscale. It is concluded that, with the reduction in grain size (less than $\sim 60$ to $68 \mathrm{~nm}$ ), the stress hysteresis loop size and temperature dependence of phase transformation stress reduce drastically, leading to the vanishing of the stress hysteresis and to the collapse of the Clausius-Clapeyron equation [21]. Also, measurement of lattice parameters and associated lattice strains shows that with reduction of grain size, the phase transformation mechanism changes into a continuous lattice deformation (inside the nano grains), as compared to the conventional nucleation and growth mode in coarse-grained polycrystals [23]. In another study by Ahadi and Sun [22] it is observed that for polycrystalline NiTi SMA, the effect of strain rate on the hysteresis loop area and transformation stress reduces with the reducing grain size and vanishes for any grain size below approximately $10 \mathrm{~nm}$. Therefore, it becomes very important to have an appropriate understanding of the nature and evolution kinetics of such internal strains, because (a) they govern the stability of different phases and (b) they can be used in constitutive modeling of the SMA as an internal variable in conjunction with the B19 variants and B2 phase fraction. These strains have been computationally studied for the B2 phase [24], but not explicitly for the B19 phase. This is mainly because the developed internal strain in the martensite variants varies with the variants. Based on crystallographic theory [25-27], it is possible to obtain the internal strain tensor of individual B19 $\square$ variants, thus, based on the phase fraction of different B19 $\square$ variants, these strain tensors can be used to obtain the total internal strain in the spatial domain of B19 $\square$. However, evolution of different phases and internal strains are intercoupled, thus, to study the entire phase transformation process (B2 $\square$ different B19 $\square$ variants) requires a large number of nonlinear simulations which is computationally challenging, at least for the atomic and mesoscale scales. The complexity increases for polycrystalline NiTi due to the presence of grain boundaries. Despite of its importance, the interplay of cell/specimen size and grain size has received little attention at fine and meso scales, perhaps because of the difficulties in addressing it. Some attempts to shed light in such interplay are reported in $[28,29]$ albeit for mechanical properties.

Along the same lines, it has been observed that due to the evolution of different variants of B19 $\square$ and B2 phases, the phase transformation process becomes inherently stochastic in nature $[1,3,8]$ and occurs hierarchically over a very wide range of spatiotemporal scales $[1,3,8,15]$. However, the ins and outs of such aspects remain poorly understood. It is worth studying and understanding such length-scale-dependent stochastic phase transformation and then linking the length scales to the propagation or transfer of stochasticity from micro and meso scales to macro/ continuum scales. Such multiscale coupling would not only provide a robust material model at the continuum level, but would also enable the incorporation of the 
effects of length scales in the evolution kinetics of the phase transformation process. In this context, review papers [30,31] and other field specific studies [32-34] provide details about computational modeling of relevant multiscale and multiphysics problems (MSMP). Waveletbased MSMP methods have received sufficient attention for addressing both spatial [8,35-37] and temporal scaling [38-41]. Further, wavelet-based techniques provide advantages over other multiscale methods because of their ability to perform scale-wise data fusion, flexibility in upscaling and downscaling of the dynamic features, and finally, capability in preserving non-stationary features in the dynamics which is lost or significantly distorted by other types of infinite basis transforms (e.g., Fourier analysis). Therefore, herein, small size NiTi samples are studied via MD simulations, and larger size samples are studied via stochastic phase field (PhF) simulations. A concurrent multiresolution (wavelet) based multiscale methodology, namely the compound wavelet method (CWM) [8,35-40] is used to link the MD and PhF simulations along spatial scales, yielding a length-wise compound representation of the thermally induced martensitic phase transformation process. Finally, the CWM method is used to feed the information on length scale effects (obtained from the MD and $\mathrm{PhF}$ simulation of NiTi single crystals), onto a phenomenological material model, which was originally developed by Zotov et al [42] based on their experimental study.

It can be expected that, at the atomic to meso scales, interaction of the different phases (B2 and B19 $\square$ ) with the free surfaces causes significant variation in the phase fraction evolution process and transformation kinetics, as briefly addressed in recent computational studies by the authors $[8,15]$. However, in those previous studies by authors $[8,15]$, as well as in some other relevant studies [11,13], the coupled effect of grain size and length scale, on the thermally induced martensitic phase transformation (MPT) was not addressed. Rather, all those previous studies $[8,11,13,15]$ mainly explore the effects of the side length (thin samples of constant thickness i.e. $2 \mathrm{~nm}$ ) or the size effect (nano particles or spheres) on the thermally induced martensitic phase transformation process for NiTi single crystal. A general spatial multiscale coupling framework is proposed by the authors [8], to transfer information from fine to coarse scales in the solid to solid phase transformation process. In that study [8], considering a single size case (i.e. for the simulation cell where the martensite phase fraction saturates), uncertainties in the evolution (due to the temperature) of B2 (austenite) phase and different B19 variants (martensite) are propagated from small scales (micro/ meso level) to coarse scales (continuum level). Thus, this study extensively broadens the results from the previous studies by the authors $[8,15]$ and in addition:

- Explicitly studies the effect of length scale (i.e. thickness and side length) on phase transformation process i.e. evolution kinetics and transformation temperatures of single crystal NiTi sample.

- For the first time, shows, computationally, the effect of length scales and temperature, on the development of internal strain in the B19 $\square$ phase.

- Performs MSMP simulations to link different length scales and transfer the statistical information of length scale effects from micro (i.e. atomic) and meso (i.e. phase field) scales to the macro (continuum) scale.

- Develops a unique and novel multiscale method for indirect modeling of the phase transformation process in polycrystalline NiTi by employing the wavelet coefficients from single crystal simulations of various size cells. 
- Employs phase field (PhF) simulations to demonstrate the interplay of grain size and length scale effect on the thermally induced martensite phase fraction evolution, for polycrystalline NiTi.

- Finally, using the MSMP coupling results, improves already existing, continuum scale, phenomenological constitutive models.

\section{Simulation methods and multiscale coupling}

The MD and PhF methods used in this study, as well as the CWM methodology for linking the results from the two simulation methods are described in detail in a very recent study by the authors [8]. Heat diffusion is modeled herein using the lattice Boltzmann method (LBM), similarly to what is used in [8]. Therefore, the brief description of those methods are provided herein. Rather, the key points that relate to the objective of the present paper are only addressed, and the paper concentrates on the results relevant to shape effects and internal strain in the B19 $\square$ phase, the strains developed in B19 $\square$ and on modeling polycrystalline samples.

\subsection{MD potential, simulation process and structural characterization}

MD simulations are performed employing the Finnis-Sinclair many body interatomic potential $[4,8,11,14,15,43]$, developed based on the embedded atom method (EAM) and considering a tight binding second moments approximation (TBSMA) theory. Quasi-static MD simulations are performed in LAMMPS [44], for temperature driven phase transformation process. Brief description of the simulation process is provided subsequently, while more details are available in recent studies by the authors $[8,15]$. This embedded-atom method (EAM) potential was initially proposed by Lai and Liu [43], based on the secondmoment approximation of tight-binding theory (TBSMA). According to this potential, the total energy of the system over all atoms is expressed as

$$
E=\sum_{i}\left\{\sum_{j \neq i} A_{\alpha \beta} \exp \left[-p_{\alpha \beta}\left(\frac{r_{i j}}{d_{\alpha \beta}}-1\right)\right]-\sqrt{\sum_{j \neq i} F\left(r_{i j}\right)}\right\}
$$

The first term in (1) indicates the pairwise interaction energy and the second term represents many-body effects. Recently the many-body interaction part (second term in (1)) of Lai and Liu potential has been modified by Zhong et al [4,45], where the second term in (1) is expressed through

$$
F\left(r_{i j}\right)=\left\{\begin{array}{r}
\xi_{\alpha \beta}^{2} \exp \left[-2 q_{\alpha \beta}\left(\frac{r_{i j}}{d_{\alpha \beta}}-1\right)\right] r_{i j} \leq r_{1} \\
c_{0, \alpha \beta}+c_{1, \alpha \beta}\left(r_{i j}-r_{1}\right)+c_{2, \alpha \beta}\left(r_{i j}-r_{1}\right)^{2}+c_{3, \alpha \beta}\left(r_{i j}-r_{1}\right)^{3} \quad r_{1} \leq r_{i j} \leq r_{c}
\end{array}\right.
$$

In (1) and (2), $i$ and $j$ denote atoms, $\alpha, \beta$ denote the type of atoms (i.e. $\mathrm{Ni}$ or Ti), and $r_{1}$ and cut-off radius $\left(r_{c}\right)$ are adopted as $4.0 \AA$ and $4.2 \AA$, respectively. Different parameters of this potential are determined through fitting the properties (i.e. lattice parameters, cohesive energy, elastic constants and vacancy formation energy) of the B2 phase at $0 \mathrm{~K}$ temperature, from first principles calculations [4]. More details about this EAM potential parameters are provided in Zhong et al [4]. 
To study length scale effects, free boundary conditions in all three directions of the simulation cell are imposed. In doing so, periodic boundary condition is used in all sides, with vacuums (empty space) all around the simulation cell are considered. In every direction, a minimum 20 $\AA$ empty space is provided, while the potential cutoff radius $\left(r_{c}\right)$ is $4.2 \AA$. This eliminates the possibility of interaction between surface atoms, due to the periodic boundaries and thus enables the capturing of length scale effects. Based on such boundary conditions, at constant temperature, at first NPT simulation, followed by NVT equilibration is performed. The size and thickness of the examined cells are described in the results and discussion section. Initially, pristine B2 crystal structures, i.e. 3-D simulation cells with the crystallographic orientation [110], [ 11 10] and [001] are considered. For the horizontal plane, directions along thickness [110] and along one side length [ 1 10] are considered. For the vertical direction, the [001] crystal orientation is considered. The simulation process is as follows. First, a pristine austenite sample is relaxed using an energy minimization method. Then the sample is subjected to a velocity distribution (at a constant temperature, controlled via proper thermostat and barostat) and held for 360 ps, under an NPT stabilization where the systems pressure is kept constant at 0 bars. The NPT stabilized sample is then subjected to NVT conditions for an additional 240 ps. The atomic coordinates were averaged over 20 ps in order to avoid effects from thermal fluctuations. In each case of NPT or NVT stabilization, the adopted time step is $\Delta t=i \quad 0.0005$ ps. During simulation, the temperature and pressure are controlled by a Nose-Hoover thermostat and Berendsen barostat. It has been verified that the simulated cells are well-equilibrated and the fluctuations in the total energy and temperature are smaller than the $0.001 \mathrm{eV} /$ atom and $0.50 \mathrm{~K}$, respectively. Finally, the well equilibrated NVT simulations (i.e. averaged over last 20 ps) is used to report the results.

To distinguish austenite B2 phase from the different martensite B19 $\square$ phase variants, the bond length based order parameter proposed by Mutter and Nielaba [8,11,12,15,46] was adopted. This order parameter has a value of -1.0 and +1.0 for pristine B2 and B19 $\square$ structures, respectively. It is expressed as

$$
\chi\left(d_{0}, d_{1}\right)=\frac{\left[d_{0}\left\{d^{(B 2)}+d_{1}^{\left(B 19^{\prime}\right)}\right\}\right]-\left[d_{1}\left(d^{(B 2)}+d_{0}^{\left(B 19^{\prime}\right)}\right\}\right]}{\left[d^{(B 2)}\left\{d_{0}^{\left(B 19^{\prime}\right)}-d_{1}^{\left(B 19^{\prime}\right)}\right\}\right]}
$$

where bond length parameters are $d^{(B 2)}=2.62 \AA, d_{0}^{\left(B 19^{\prime}\right)}=2.46 \AA \wedge d_{1}^{\left(B 19^{\prime}\right)}=2.65 \AA$. The order parameter becomes +1.0 for the martensite B19 $\square$ phase when $d_{0}=d_{0}^{\left(B_{19}^{\prime}\right)}$ and $d_{1}=d_{1}^{\left(B 19^{\prime}\right)}$. For the austenite B2 phase $d_{0}=d_{1}=d^{(B 2)}$ and thus the value of the order parameter becomes -1.0. However, the lattice parameters of B2 and B19 $\square$ phases change due to the linear thermal expansion or contraction $(\alpha \Delta T)$, and due to the internal strains $\left(\varepsilon^{i}\right)$ that develop during the phase transformation process. Thus the order parameter shows a narrow Gaussian distribution around -1.0 and +1.0 for the B2 and B19 $\square$ phases, respectively $[8,15,24]$. More details about this order parameters can be found in $[8,11,12,15,24]$, and in the supplementary material.

\subsection{Phase field (PhF) simulation: model and method}

Phase field $(\mathrm{PhF})$ simulations, a widely used and validated methodology [7,16,24], is employed to simulate the martensite phase fraction evolution, based on the Ginzburg-Landau 
(GL) type polynomial energy function [7,8]. In PhF simulations, austenite B2 transforms into 12 different variants of monoclinic martensite B19๑, with all field variables evolving stochastically over the simulation domain. The growth or evolution rate of the $i^{\text {th }}$ field variable $\psi_{i}$ can be expressed through the time dependent Ginzburg-Landau (TDGL) kinetic equation as $[7,8]$

$$
\frac{\partial \psi_{i}}{\partial t}=\sum_{j=1}^{12}\left[\dot{\vartheta}_{i j}\left(G_{j}^{\text {local }}+G_{j}^{\text {gradient }}+G_{j}^{\text {elastic }}\right)\right]+\eta_{i}(x, t)
$$

where $\dot{\vartheta}_{i j}$ is the kinetic coefficients matrix, $G_{j}^{\text {local }} ; G_{j}^{\text {gradient }}$ and $G_{j}^{\text {elastic }}$ represent the driving force acting on the $j^{\text {th }}$ field variable due to the local free energy density $\left(g^{\text {local }}\right)$, gradient energy density $\left(g^{\text {gradient }}\right)$ and elastic energy density $\left(g^{\text {elastic }}\right)$, respectively, and $\eta_{i}(x, t)$ is the Langevin noise term to incorporate the stochasticity in the evolution of the B19 $\square$ variants.

For $i \neq j$, the driving force on the $i^{\text {th }}$ phase field variable is considered that it does not affect the evolution of $j^{\text {th }}$ phase field variable, thus $\dot{v}_{i j}=\vartheta \delta_{i j}$, where $\delta_{i j}$ is the Kronecker delta. Further, from the fluctuation-dissipation theorem [7], the correlation function of the Langevin noise term in the spatiotemporal domain can be expressed as a function of temperature $\left\langle\eta_{i}(x, t) \eta_{i}(x+s, t+\tau)\right\rangle=2 k_{B} T \dot{\vartheta}_{i j} \delta_{i j} \delta(s) \delta(t)$, where $k_{B}$ is the Boltzmann constant, $T$ is simulation temperature and $\delta(\cdot)$ is the Dirac delta function. With this, (4) results into

$$
\frac{\partial \psi_{i}}{\partial t}=\vartheta \sum_{i=1}^{12}\left[\left(G_{i}^{\text {local }}+G_{i}^{\text {gradient }}+G_{i}^{\text {elastic }}\right)\right]+\eta_{i}(x, t)
$$

Next, the energy density functions can be expressed as [7,8]

$$
\begin{aligned}
g^{\text {local }}=g_{0}+\Delta g(T)\left(\frac{A}{2}\left(\sum_{i=1}^{12} \psi_{i}^{2}\right)\right. & \left.-\frac{B}{3}\left(\sum_{i=1}^{12} \psi_{i}^{3}\right)+\frac{C}{4}\left(\sum_{i=1}^{12} \psi_{i}^{2}\right)^{2}+\frac{D}{4}\left(\sum_{i=1}^{12} \psi_{i}^{4}\right)\right\} \\
g^{\text {gradient }} & =\frac{1}{2} \sum_{i=1}^{12} \beta_{m n}\left(\psi_{i}\right)\left(\frac{\partial \psi_{i}}{\partial x_{m}}\right)\left(\frac{\partial \psi_{i}}{\partial x_{n}}\right) \\
g^{\text {elastic }} & =\frac{1}{2}\left(\varepsilon-\varepsilon^{i}\right)^{T} C\left(\varepsilon-\varepsilon^{i}\right)
\end{aligned}
$$

In (6.a), $\quad g_{0}$ represents the free energy density of the austenite phase, $\Delta g(T)$ the difference in the free energy between austenite and martensite phase at temperature $T$, and $A, B, C \wedge D$ are constants that control the shape of the free energy function. In (5.b), for isotropic gradient energy coefficient $\beta_{m n}\left(\psi_{i}\right)$ is assumed the same for all the field variables, i.e. $\quad \beta_{m n}\left(\psi_{i}\right)=\beta \delta_{m n}$ and thus (6.b) will can be rewritten as $[7,8]$

$$
g^{\text {gradient }}=\frac{1}{2} \sum_{i=1}^{12} \beta \delta_{m n}\left(\frac{\partial \psi_{i}}{\partial x_{m}}\right)\left(\frac{\partial \psi_{i}}{\partial x_{n}}\right)=\frac{\beta}{2} \sum_{i=1}^{12}\left(\frac{\partial \psi_{i}}{\partial x_{m}}\right)^{2}=\frac{\beta}{2} \sum_{i=1}^{12}\left(\nabla \psi_{i}\right)^{2}
$$

In (6.c), $\varepsilon$ is the linearized strain tensor, i.e. $\left\{\varepsilon_{11}, \varepsilon_{22}, \varepsilon_{33}, 2 \varepsilon_{23}, 2 \varepsilon_{31}, 2 \varepsilon_{12}\right\}^{T} ; \quad \varepsilon^{i}$ is the nonlinear stress-free strain due local phase transformation, i.e. 
$\left\{\varepsilon_{11}^{i}, \varepsilon_{22}^{i}, \varepsilon_{33}^{i}, 2 \varepsilon_{23}^{i}, 2 \varepsilon_{31}^{i}, 2 \varepsilon_{12}^{i}\right\}^{T}$, and $C$ is the $6 \times 6$ isotropic elasticity tensor in the Voigt notation system [7,8]. Elastic interactions play a critical role in the evolution of martensite phase fraction, thus, the accurate estimation of the elastic energy density is important. Since in the present study no external strain is applied (i.e. $\varepsilon=0$ ), (6.c) can be written as $[7,8]$

$$
g^{\text {elastic }}=\frac{1}{2}\left(\varepsilon^{\zeta}\right)^{T} C\left(\varepsilon^{i}\right)=\frac{1}{2}\left(\sum_{i=1}^{12} \psi_{i} \cdot \varepsilon_{i}^{0}\right)^{T} C\left(\sum_{i=1}^{12} \psi_{i} \cdot \varepsilon_{i}^{0}\right)
$$

where $\varepsilon_{i}^{0}$ represents the transformation strain tensor associated with the $i^{\text {th }}$ phase field variable [7,8]. More details about the estimation of $\varepsilon_{i}^{0} \quad$ is provided in the supplementary material. Here, $\varepsilon^{i}$ represents the stress-free strain (i.e. internal strain tensor in B19 $)$ ), which arises due to local martensitic phase transformation from B2 to B19

$$
\varepsilon^{i}=\sum_{i=1}^{12} \psi_{i} \cdot \varepsilon_{i}^{0}
$$

Therefore, the associated driving forces for the evolution of $j^{\text {th }}$ field variable can be described through the following set of equations

$$
\begin{gathered}
G_{i}^{\text {local }}=\frac{-\partial g^{\text {local }}}{\partial \psi_{i}}=\Delta g(T)\left\{-A \psi_{i}+B \psi_{i}^{2}-C \psi_{i}\left(\sum_{j=1}^{12} \psi_{j}^{2}\right)-D \psi_{i}^{3}\right\} \\
G_{i}^{\text {gradient }}=\frac{\partial g^{\text {gradient }}}{\partial \psi_{i}}=\beta\left(\nabla^{2} \psi_{i}\right) \\
G_{i}^{\text {elastic }}=\frac{-\partial g^{\text {elastic }}}{\partial \psi_{i}}=-\left[\sum_{i=1}^{12} \psi_{i} \cdot\left(\varepsilon_{i}^{0}\right)^{T}\right] \cdot\left(C^{T} \varepsilon_{i}^{0}\right)=F_{i}^{e l}
\end{gathered}
$$

With all the terms in TDGL kinetic equation in (5) are obtained, the stochastic spatiotemporal evolution of any field variable (e.g. the $j^{\text {th }}$ field variable) can be expressed as $[7,8]$

$$
\frac{\partial \psi_{i}}{\partial t}=\vartheta\left[\Delta g(T)\left\{-A \psi_{i}+B \psi_{i}^{2}-C \psi_{i}\left(\sum_{j=1}^{12} \psi_{j}^{2}\right)-D \psi_{i}^{3}\right\}+\beta\left(\nabla^{2} \psi_{i}\right)+F_{i}^{e l}\right]+\eta_{i}(x, t)
$$

Equation (11) is easily normalized in terms of length and time scale. By employing evenly spaced grid size $(\Delta x)$ and time steps $(\Delta t)$ in (11), it follows that

$$
\frac{\partial \psi_{i}}{\partial \tilde{t}}=\left[\left\{-A \psi_{i}+B \psi_{i}^{2}-C \psi_{i}\left(\sum_{j=1}^{12} \psi_{j}^{2}\right)-D \psi_{i}^{3}\right\}+\widetilde{\beta}\left(\widetilde{\nabla}^{2} \psi_{i}\right)+\frac{F_{i}^{e l}}{\Delta g(T)}\right]+\tilde{\eta}_{i}(\tilde{x}, \tilde{t})
$$

where dimensionless time and time steps are expressed in terms of real time $t$ and time steps $\Delta t$, i.e. $\tilde{t}=t \vartheta \Delta g(T)$ and $\Delta \tilde{t}=\Delta t \vartheta \Delta g(T)$, and the dimensionless spatial coordinate system is expressed in terms of the grid size $\Delta x$ i.e. $\tilde{x}=x / \Delta x$, and $\widetilde{\beta}=\beta / \Delta g(T)(\Delta x)^{2} \quad$ denotes the normalized isotropic gradient energy coefficient. Finally, the normalized Langevin noise term $\tilde{\eta}_{i}(\tilde{x}, \tilde{t})$ is generated as a random number from the spatiotemporally uncorrelated normal distribution with zero mean and variance of $2 k_{B} T / \Delta g(T)(\Delta x)^{3}$. 
For the simulation process, initially 2-D B2 simulation cells of different sizes (from $32 \mathrm{~nm}$ to $2048 \mathrm{~nm}$ ) are considered. The crystallographic orientation of the B2 simulation cells are adopted as [110] (along $\mathrm{x}$ direction) and [001] along (y direction) and then the simulation cells are stabilized at constant temperature, as detailed subsequently. The spatial and temporal resolutions are adopted as $\Delta x=1 \mathrm{~nm}$ and $\Delta t=0.001 \mathrm{~ns}$, respectively, and simulations are performed for a total time of $20 n s$, out of which, for the first $5 n s$, the simulation is perturbed by uncorrelated Langevin noise. Convergence of the simulation process is ensured by comparing different statistical measures of the martensite phase fraction between two consecutive iteration steps, i.e. iteration is stopped once $\Delta_{Y} \leq \delta_{Y}$. Here, for any variable $Y$, the difference in value between two consecutive iteration steps is expressed as $\left.\Delta_{Y}=\|\left(Y_{n+1}-Y_{n}\right) / Y_{n}\right\} \mid$ and the tolerance limit is adopted as $\delta_{Y}=0.001$. Further details about the PhF simulation process is provided in [8].

\subsection{Lattice Boltzmann method (LBM): heat transfer}

Heat transfer in the simulation domain is modeled with the lattice Boltzmann method (LBM) that has received significant attention in simulation of transport processes [47-53]. Considering an isothermal process for heat transfer, Peng's implementation of thermal LBM [48] is a simplified form of He et al [47] thermal LBM. It considers that heat diffuses through the NiTi medium, and heat is dissipated during phase transformation. For the transient case, the heat diffusion equation with energy dissipation can be expressed as

$$
\frac{\partial T}{\partial t}=\left(\frac{1}{\rho c_{p}}\right)[\{\nabla \cdot(k \nabla \cdot T)\}-\dot{Q}]
$$

where $\rho, c_{p}, k$, and $\dot{Q}$ are the density, thermal conductivity, heat capacity and heat energy dissipation, respectively.

Here, the LBM solves the generalized heat diffusion equation via propagation and collision steps, employing specific so-called elements [8,51-53]. In the present study, the D2Q9 lattice configuration is used for the transport process [8,51-53]. The temperature dependent thermal conductivity, heat capacity and heat energy dissipation are obtained from different experimental studies [54-56] and further details are available in [8]. In the LBM framework for heat transfer through a solid, all velocity components are zero, i.e. $u=0$. It is considered that during the heat diffusion process, heat energy dissipates due to phase transformation, thus, for the transient condition, the generalized heat diffusion equation solved by LBM contains a heat source term. In two dimensions (2-D), both mass and heat transport can be described by the lattice Boltzmann equation, expressed as [8,47-53]

$$
\begin{gathered}
f_{\alpha}\left(x+e_{\alpha} \delta_{t}, t+\delta_{t}\right)=\left(1-\frac{1}{\tau_{f}}\right) f_{\alpha}(x, t)+\left(\frac{1}{\tau_{f}}\right) f_{\alpha}^{e q}(\rho, u) \\
g_{\alpha}\left(x+e_{\alpha} \delta_{t}, t+\delta_{t}\right)=\left(1-\frac{1}{\tau_{g}}\right) g_{\alpha}(x, t)+\left(\frac{1}{\tau_{g}}\right) g_{\alpha}^{e q}(T, u)-\varpi_{\alpha}\left(1-\frac{1}{2 \tau_{g}}\right) \dot{Q}\left(\frac{1}{\rho c_{p}}\right)
\end{gathered}
$$

where $x$ the space coordinates or position vector; $e_{\alpha}$ the discrete velocity components, $u$ the velocity vector; $\delta_{x}=\delta_{y} \quad$ the lattice constant (grid size)/ spatial resolution; $\delta_{t}$ 
the time step/ temporal resolution; $\tau_{f}=\left(\frac{3 v}{c \delta_{x}}+\frac{1}{2}\right)$ and $\tau_{g}=\left(\frac{3 k}{2 c \delta_{x} \rho c_{p}}+\frac{1}{2}\right)$ are the dimensionless relaxation time for mass transport and heat energy transfer, respectively, $u$ is the kinematic viscosity of the medium, $c$ the speed in the lattice that depends on the spatial and temporal resolution, expressed as $c=\delta_{x} / \delta_{t}$ and is related to the speed of sound in the lattice through $c_{s}^{2}=R T=\frac{c^{2}}{3}$, where $R$ is the universal gas constant and $T$ is absolute temperature of the system. Further, $f_{\alpha}$ and $g_{\alpha}$ denote the density and heat energy distribution function, respectively, $f_{\alpha}^{e q}$ and $g_{\alpha}^{e q}$ denote the equilibrium density and the heat energy distribution function, respectively, and $e_{\alpha}$ is the discrete velocities components for the D2Q9 element (as shown in Fig. S4 of supplementary material). More details about those quantities is provided in the supplementary material.

Finally, the density, velocity and temperature at any node are calculated by [8]

$$
\rho(x, t)=\sum_{\alpha} f_{\alpha}(x, t)
$$

$$
u=\frac{\sum_{\alpha} e_{\alpha} f_{\alpha}(x, t)}{\sum_{\alpha} f_{\alpha}(x, t)}
$$

$$
T(x, t)=\sum_{\alpha} g_{\alpha}(x, t)-\dot{Q}\left(\frac{\delta_{t}}{2 \rho c_{p}}\right)
$$

\subsection{Multiscale coupling of phase transformation process: CWM method}

In this study an important predictive feature is added to the compound wavelet matrix (CWM) method, mainly based on the recent study by Gur et al $[8,41]$ on the wavelet based surrogate time-series generation for heterogeneous catalysis. The following 1-D example illustrates the methodology $[8,41]$.

Considering a 1-D discrete data vector $y(x)$ of length $n=2^{N}$, the wavelet transform of $y(x)$ hierarchically decomposes $y(x)$ into $N\left(\dot{i} \log _{2}(n)-2^{2}\right)$ number of scales and returns $2^{N}$ wavelet transform (WT) coefficients. The first $2^{N-1}$ WT coefficients represent the finest characteristics of the original signal, the next $2^{N-2}$ WT coefficients represent the next level (coarser) information in the data and so on. This process is well documented in the literature [8,37,39-41]. Thus, the wavelet transform of $y(x)$ will yield $W_{y}(s, x)$, i.e. a set of wavelet coefficients, where $s$ represents scale and $x$ stands for spatial coordinate in the fine scale simulation. Now, $W_{y}(s, x)$ is decomposed as

$$
W_{y}(s, x)=\phi_{y}\left(s_{0}, \Delta x\right) \bigoplus \phi_{y}\left(s_{1}, \Delta x\right) \bigoplus \phi_{y}\left(s_{2}, 2^{1} \Delta x\right) \bigoplus \cdots \cdots \oplus \phi_{y}\left(s_{N}, 2^{N-1} \Delta x\right)
$$


where $\oplus$ denotes scale wise association in the wavelet formalism, $\phi_{y}\left(s_{i}, 2^{i-1} \Delta x\right)$, $i=1,2, \cdots, N$ denotes the wavelet transform at scale $s_{i}$ with the scaling interval $2^{i-1} \Delta x$ and $\phi_{y}\left(s_{0}, \Delta x\right)$ represents the wavelet transform at the coarsest scale in fine scale simulation for a particular scaling function.

Base on the wavelet transform, the multiscale coupling of martensite phase fraction evolution is performed via the CWM method, as discussed subsequently. If, for thermally induced martensite phase transformation the martensite phase fraction, $\xi$, is only available at specific and slightly overlapping wavelet space scales, e.g. $\xi(x)$ at fine MD territory scales, $a_{\text {fine }}$, and $\xi(X)$ at coarse PhF territory scales, $a_{\text {coarse }}$, given the wavelet transform of $\xi$ at fine scales, $W_{\xi}\left(a_{\text {fine }}, x\right)$, and that at coarse scales, $W_{\xi}\left(a_{\text {coarse }}, X\right)$, with the $x$ and $X$ denoting the location in MD and PhF domain, then the wavelet synthesis or fusion process, implies that

$$
W_{\xi_{\text {cum }}}(a, X)=W_{\xi}\left(a_{\text {fine }}, x\right) \cup W_{\xi}\left(a_{\text {coarse }}, X\right)
$$

i.e. the union (with proper weights for overlapping scales) of the wavelet transform at fine and coarse scales. Next, the inverse wavelet transform ( IWT ) is performed on the compounded wavelet coefficients, to obtain the martensite phase fraction vector after compounding, thus

$$
\xi_{c w m}(X)=I W T\left[W_{\xi_{\text {cum }}}(a, X)\right]=I W T\left[W_{\xi}\left(a_{\text {fine }}, x\right) \cup W_{\xi}\left(a_{\text {coarse }}, X\right)\right]
$$

The CWM multiscaling process was described above for simplicity in 1-D, yet its extension to 2-D is straightforward. In the 2-D case, both coarse and fine scale simulations are performed in 2-D, and so is the forward and inverse wavelet transform.

Table-1: Algorithm of multiscale coupling framework via CWM method

1. Given the coarse and fine martensite phase fraction (MPF):

$$
\begin{aligned}
& \xi_{\text {coarse }}(X) \text { with } X \in\left[X_{n}, X_{n}+\Delta X\right] \text { and } \xi_{\text {fine }}(x) \text { with } x \in\left[X_{n}, X_{n}+x\right] \text {; where } \\
& X \ll \Delta X
\end{aligned}
$$

2. Compute wavelet transforms (WT) of MPF:

$$
W_{\xi_{\text {coarse }}}(s, X)=W\left[\xi_{\text {coarse }}(X)\right] \text { and } W_{\xi_{\text {fine }}}(s, x)=W\left[\xi_{\text {fine }}(x)\right]
$$

3. Apply window filter on the wavelet coefficients of MPF:

$$
W_{\xi_{\text {couse }}}^{H_{a, b}}(s, X)=H_{(a, b)} \cdot W_{\xi_{\text {course }}}(s, X) \text { and } \quad W_{\xi_{\text {fine }}}^{H_{b, c \mid}}(s, X)=H_{(b, c)} \cdot W_{\xi_{\text {fine }}}(s, X)
$$

4. Compute compounding i.e. the coefficient of compound wavelet matrix (CWM):

$$
W_{\xi_{\text {cum }}}(s, X)=W_{\xi_{\text {coarse }} \oplus \text { fine }}^{H_{a, c)}}(s, X)=W_{\xi_{\text {coarse }}}^{H_{a, b}}(s, X) \bigoplus W_{\xi_{\text {fine }}}^{H_{b, c}}(s, X)
$$

5. Compute inverse wavelet transform (IWT) to obtain compounded MPF:

$$
\xi_{c w m}(s, X)=C W M\left(\xi_{\text {coarse }}(X), \xi_{\text {fine }}(x)\right)=W^{-1}\left[W_{\xi_{\text {cum }}}(s, X)\right]
$$

The relevant flow chart and more details about the CWM method with the component for predicting the fine scale wavelet coefficients is provided in [8]. However, the algorithm is provided here in Table -1, showing the main relevant steps of the multiscale coupling method, where $W$ and $W^{-1}$ denote the wavelet transforms (WT) and the inverse wavelet transforms (IWT), respectively, and $H_{\left(s_{1}, s_{2}\right)}$ and refers to the Heaviside function defined as 


$$
H_{\left(s_{1}, s_{2}\right)}(s)=\left\{\begin{array}{l}
1 \text { if } s_{1}<s<s_{2} \\
0 \text { otherwise }
\end{array}\right.
$$

Also, $a$ represents the coarsest scale resolved by the $\mathrm{PhF}$ simulation method, $c$ the smallest scale resolved by the MD simulation method and $b$ the cutoff scale, chosen based on the dominant scales resolved by the coarse and fine (by comparing the energy at the different wavelet scales). For more details about the CWM based multiscale coupling method, we refer to $[8,35-41]$.

\section{Evolution kinetics and material modeling}

This section provides a brief description of the temperature dependent martensite phase fraction evolution kinetics, phase transformation temperatures (martensite and austenite start and finish), and the thermo-mechanical material model of polycrystalline NiTi SMA at continuum level.

\subsection{Phase fraction evolution}

Phenomenological models based on extensive continuum level experimental data on thermally induced martensitic phase fraction have been proposed [8,15,42,57-59]. Experiments are reported on annealed (at a temperature of $750{ }^{\circ} \mathrm{C}$ ) polycrystalline austenite NiTi SMA (mainly [110] B2 crystal with weak [ 1 1 11] B2 crystal), with cooling and heating rate of $20{ }^{\circ} \mathrm{C} / \mathrm{min}$, under various concentrations of $\mathrm{Ni}$ and $\mathrm{Ti}$ [42]. The model considered herein, essentially captures the kinetics of phase transformation rate as a function of phase fraction. It is assumed that, (a) the austenite transformation rate at any given temperature is proportional to the nucleation rate of austenite per unit volume and (b) the size of the austenite nucleus increases linearly with the austenite phase fraction. According to the model, the temperature dependent austenite phase fraction evolution is described through the Richards equation [42], i.e.

$$
\xi(T)=1-\left[1+\exp \left\{-g \cdot v\left(T-T_{m}\right)\right\}\right]^{-1 / v)}
$$

where $\xi(T)$ is the temperature dependent austenite phase fraction, $T$ the temperature of interest in $\mathrm{K}, T_{m}(\dot{\zeta} K)$ the temperature corresponding to the maximum transformation rate, while $g\left(i K^{-1}\right)$ and $v$ (unitless) are the fit parameters which dictate the transformation or growth rate of the austenite phase. Equation (20) is adopted here to represent the martensitic phase fraction evolution as a function of temperature at coarse scales or continuum level. More details about this phenomenological model are available in $[8,15,42,57-59]$.

\subsection{Thermo-mechanical continuum material model}

The shape memory effect and super-elasticity are two paramount properties of SMAs, controlled by the thermo-mechanically induced austenite to martensite (and vice versa) phase transformation process. Above the austenite finish temperature $A_{f}$ the SMA is in the austenite phase and upon loading it transforms into martensite and during unloading the martensite back transforms into austenite. Below $A_{f}$, depending on the temperature, the SMA leaves residual strain upon unloading which can be recovered by heating above $A_{f}$. In the process of loading-unloading, the SMA dissipates a large amount of energy through its 
flag-shaped hysteresis loop which again depends on the temperature and strain rate. Here the SMA material model of Helm and Haupt [60] is adopted, which has been verified experimentally and captures all essential aspects of the SMA behavior. The transformation temperatures considered here are those of NiTi SMA: (i) for the martensite start $M_{s}$ and finish $\quad M_{f}$ temperatures are 295 and $275 \mathrm{~K}$, respectively; (ii) for the austenite start $A_{s}$ and finish $A_{f}$ temperatures are 310 and $335 \mathrm{~K}$, respectively. For uniaxial stress, it is expressed as

$$
\sigma=F_{1}\left(\varepsilon, \varepsilon^{i}, T\right)
$$

$$
\dot{\varepsilon}^{i}=F_{2}\left(\sigma, \sigma^{i}, \xi, T\right)
$$

$$
\sigma^{i}=F_{3}\left(\sigma, \sigma^{i}, \xi, \dot{\xi}, T\right)
$$

$$
\xi=F_{4}\left(\varepsilon^{i}, \dot{\varepsilon}^{i}, T\right)
$$

$$
\dot{\theta}=F_{5}\left(\sigma, \varepsilon, \dot{\sigma}^{i}, \dot{\varepsilon}^{i}, \xi, T\right)
$$

where, $\sigma$ is the developed stress, which depend on applied strain $\varepsilon$, ambient temperature $T$, developed internal strain is $\varepsilon^{i}$. Furthermore, $\varepsilon^{i}$ is the developed internal strain rate, $\sigma^{\dot{b}}$ and $\dot{\sigma}^{\dot{b}}$ are the developed internal stress and its rate, $\xi$ and $\dot{\xi}$ are the martensite phase fraction and its time rate and finally $\dot{T}$ is the time rate of temperature evolution. Thus, the material model is expressed as a system of strongly nonlinear equations that need to be solved iteratively at every time step. For detailed description of the SMA material model and the iterative solution algorithm of those system of nonlinear equations i.e. Eq. 23. (a)-(e), the reader is referred elsewhere $[8,46,60]$. The hysteretic energy dissipation capacity of SMAs and its temperature modulation is addressed below.

\section{Results and discussions}

Previous experimental $[9,10,17-19]$ as well as computational [8,11,13-15] studies at the nanometer scale demonstrate rather significant size effects on phase transformation kinetics of NiTi SMA. This section discusses the effect of length scale (shape and size) on the thermally induced phase evolution kinetics of NiTi thin samples. The effects of shape and size on the thermally induced phase fraction evolution kinetics is shown. Results at different length scale provide the basis for the multiscale coupling methodology of phase evolution kinetics in NiTi SMA. Next, the multiscale coupling results are used to improve the existing continuum material model of NiTi SMA.

\subsection{Length scale effect on phase transformation kinetics for single crystals}

Figure-1 shows MD simulations results on the effects of side length $(s)$ and thickness $(t)$ of NiTi thin samples on the thermally induced martensite phase fraction. To demonstrate the effect of side length $s$, the thickness of NiTi thin samples are kept constant 
i.e. for thickness $t=8 \mathrm{~nm}$. To show the effect of thickness $t$, the side of the NiTi thin samples is given a constant value i.e. $s=8 \mathrm{~nm}$. For each case, both of the austenitic ( $M \rightarrow A$, i.e. heating cycle) and martensitic ( $A \rightarrow M$, i.e. cooling cycle) thermally induced phase transformation process is shown, where, $M$ and $A$ are used to abbreviate the martensite and austenite phase, respectively. Fig. 1 (a) shows the effect of side length of NiTi thin samples on the temperature driven martensite phase fraction curves for $s=2,16 \wedge 64 \mathrm{~nm}$. Similarly, Fig. 1 (b) shows the effect of NiTi sample thickness on martensite phase fraction evolution, for thickness $t=2,16 \wedge 64 \mathrm{~nm}$ with constant side length. It can be observed that during austenitic phase transformation, the effect of the simulation cell side length is almost the same for both the austenite start $\left(A_{s}\right)$ and finish $\left(A_{f}\right)$ temperatures, while in the martensitic phase transformation process, this effect is more pronounced in the martensite finish temperature $\left(M_{f}\right)$ than in the start temperature $\left(M_{s}\right)$. However, change in sample thickness causes a similar level of shift in the transformation temperatures (i.e. austenite and martensite start and finish).
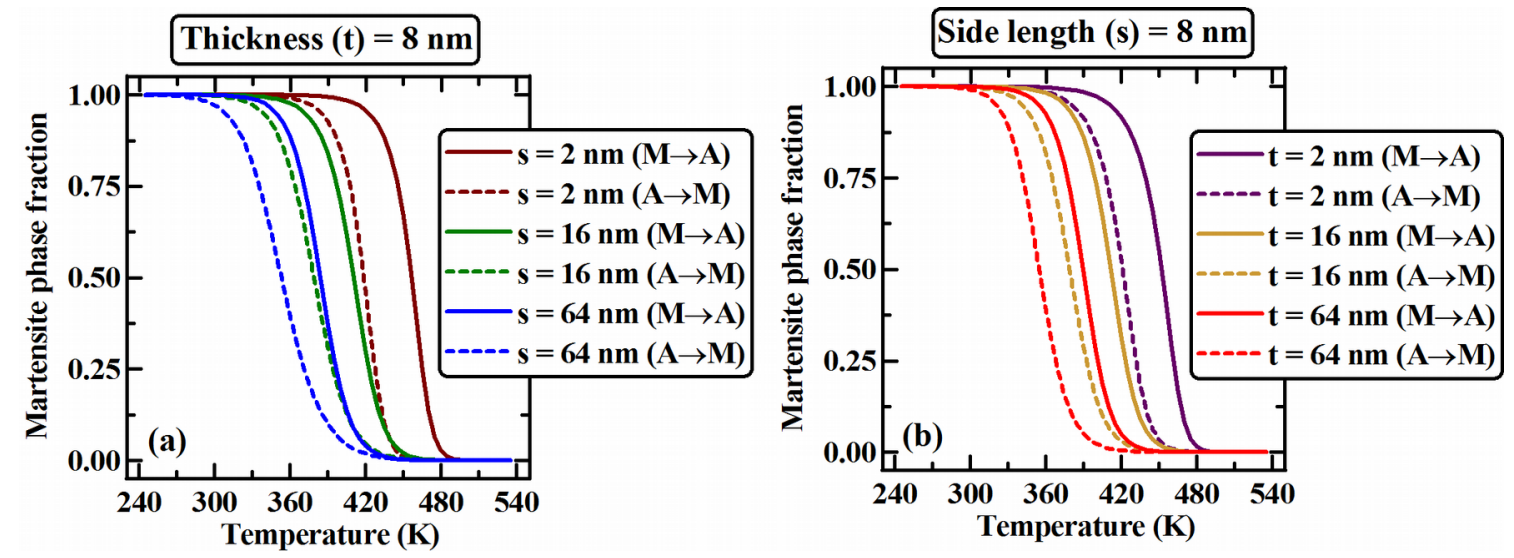

Figure-1: Effect of NiTi thin samples side length and thickness on the evolution of thermally induced martensite phase fraction (a) for constant thickness $t=8 \mathrm{~nm}$ with side length $s=2,16,64 \mathrm{~nm}$ and (b) for constant side length $s=8 \mathrm{~nm}$ with thickness $t=2,16,64 \mathrm{~nm}$.

Size effects are shown in Figure-2, where both the side length and thickness of NiTi thin samples are changed proportionally, i.e. $s=t$ and all three dimensions are increased proportionally from $2 \mathrm{nmi64nm}$. Both heating and cooling cycles (i.e. austenitic and martensitic phase transformation process) are shown. Fig. 2 (a) shows the thermally induced martensite phase fraction evolution curves for $s=t=2,4,8 \mathrm{~nm}$, and Fig. 2 (b) shows the same curves for $s=t=16,32,64 \mathrm{~nm}$. It can be observed, at any temperature, with increasing size the martensite phase fraction value decreases. Furthermore, the austenite start $\left(A_{s}\right)$ and martensite finish $\left(M_{f}\right)$ temperatures show more sensitivity to size than the austenite finish $\left(A_{f}\right)$ and martensite start $\left(M_{s}\right)$ temperatures. A discussion about the shape or the size effects in terms of the thermodynamics and available free surfaces is provided subsequently. 

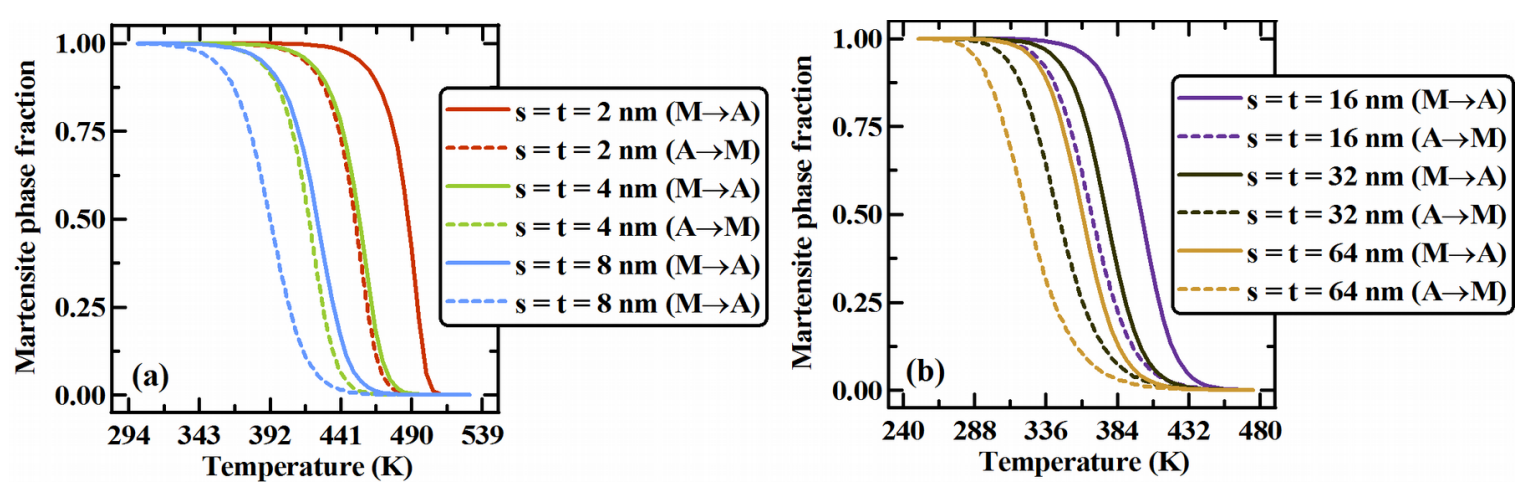

Figure-2: Size effect (variation of both thickness and side length) on the evolution of thermally induced martensite phase fraction in case of NiTi thin $\mathrm{f}$ samples (a) for $s=t=2,4,8 \mathrm{~nm}$ and (b) for $s=t=16,32,64 \mathrm{~nm}$.

As discussed here, the length scale effects are attributed to the presence of the free surfaces, which are defects. The available free surface area per unit volume can be expressed as $\gamma=A / V=(2 / t)+(4 / s)$. Thus for any fixed thickness $t$ or side length $s$, the ratio of available free surface area per unit volume is $\gamma \propto 1 / s$ or $\gamma \propto 1 / t$, with increasing $s$ or $t$ this ratio quantity decreases inversely proportional to $\sqrt{s}$ or $\sqrt{t}$, i.e. $d \gamma / d s \alpha-1 / s^{2}$ or $d \gamma / d s \alpha-1 / t^{2}$. The presence of free surfaces changes the free energy density (energy per unit volume) of phase transformation. Assuming that energy reduces due to the presence of free surface area as $e_{s}$ (per unit surface), the drop in the free energy density is $\{(2 / t)+(4 / s)\} e_{s}$ (per unit volume). Thus, increase in $s$ or $t$, decreases the free energy density, which finally increases the total energy of the system. Therefore, to obtain similar level of phase transformation, more driving force is required with increasing

$s$ or $t$. In terms of thermodynamics, with increasing $s$ or $t$, more temperature drop is required to obtain similar level of phase fraction. This ultimately shifts the phase evolution kinetics curves more towards low temperature and reduces the transformation temperature with increasing $s$ or $t$.

Similarly to temperature dependent phase fraction evolution, length scales have a strong effect on the transformation temperatures (i.e. $A_{s}, A_{f}, T_{m A}, M_{s}, M_{f}$ and $T_{m M}$ ), as shown in Figure-3. Fig. 3 (a1)-(c1) show the transformation temperatures associated with the austenitic (heating cycle) phase transformation process, while Figs. 3 (a2)-(c2) shows the ones associated with the martensitic phase transformation process i.e. for cooling cycle. In every case, with increasing side length and thickness of the NiTi thin sample, the transformation temperature decreases. Fig. 3 (d) shows the effect of NiTi thin samples thickness and side length on the width of thermal hysteresis between the austenitic and martensitic phase transformation process, which represent the difference of temperature between the heating and cooling cycle for the same martensite phase fraction. Interestingly, the width of the thermal cycle initially decreases with increasing thickness for samples of small side length. However, with increasing side length, this trend changes, i.e. the width of the thermal cycle initially decreases and after a critical thickness it increases. In every case, solid lines represent the shape effect, where only the side length changes while keeping the thickness constant. Whereas, the dotted line represents size effects, where both the side length and thickness of thin samples change. A nonlinear least-square minimization process on the data in Figs. 3 (a1)-(c2) (through MATLAB 2014) shows a power law variation for the transformation temperatures with respect to side length. Thus, all of the phase transformation 
temperatures decrease with increasing side length, following a certain power law and the power law constant and exponent shows logarithmic variation with thickness i.e. they decrease logarithmically with increasing thickness. In general, the transformation temperature model can be expressed as (i.e. as a function of $s$ and $t$ )

$$
\frac{\theta_{T}(s, t)}{\theta_{\text {bulk }}}=\delta_{c} \ln \left(\frac{t_{c}}{t}\right)[s]^{-\left\{\delta_{e} \ln \left(\frac{t_{e}}{t}\right)\right\}}
$$

where $\delta_{c}$ (in $\mathrm{nm}$ ) and $t_{c}$ (in $\mathrm{nm}$ ) are constants, respectively, and $\delta_{e}$ (unitless) and $t_{e}$ (in nm) are constant for the power law exponent. Here, $\theta_{T}(s, t)$ represents the shape and size dependency of the transformation temperatures i.e. $A_{s}, A_{f}, T_{m A}, M_{s}$, $M_{f}$ and $T_{m M}$; and $\theta_{\text {bulk }}$ represent the transformation temperatures associated with the bulk.

A similar power law model of phase transformation temperature as the function of size has been proposed previously for other cases of nanostructures (such as for nanoparticles) $[11,13]$. Those models, and the one proposed here, are only applicable at certain range of length scales, because any size below a critical length scale does not show phase transformation, as reported in [9-11,13,17-19]. In this context, Fu et al [9] reported that the growth of the surface oxide and its interfacial diffusion exert dominant constraining effects that produce high residual stress and low recovery capability. Experimental studies by Waitz et al [10,17-19] show the effect of grain size and interaction of grain boundaries on the martensite phase transformation process due to the presence of nanograins for polycrystalline samples. The presence of grain boundaries and the interaction between grains play an important role in the evolution (nucleation and growth) and stabilization of different phases (B2 and B19 $\square$ variants). In contrast to those studies, the present study, mainly shows results for single crystals (with crystal orientation of [110], [ 1 10], [001]) and thus any effect due to the residual stress or due the interaction between different crystals is not considered. However, to address the effect of grain size, grain boundaries, interaction between different grains and with the simulation cell size, phase field $(\mathrm{PhF})$ simulations for polycrystalline $\mathrm{NiTi}$ are performed. Also, an indirect method (based on the multiscale approach) is proposed to mimic the phase fraction evolution kinetics in polycrystalline NiTi. Here, the simulation results from different size single crystals i.e. the wavelet coefficients of MPF, for crystal orientation of [100], [110] and [111] are used in a unique way to reproduce the effects of polycrystals. Details about this are provided in section 4.4 , for the polycrystalline NiTi case. 

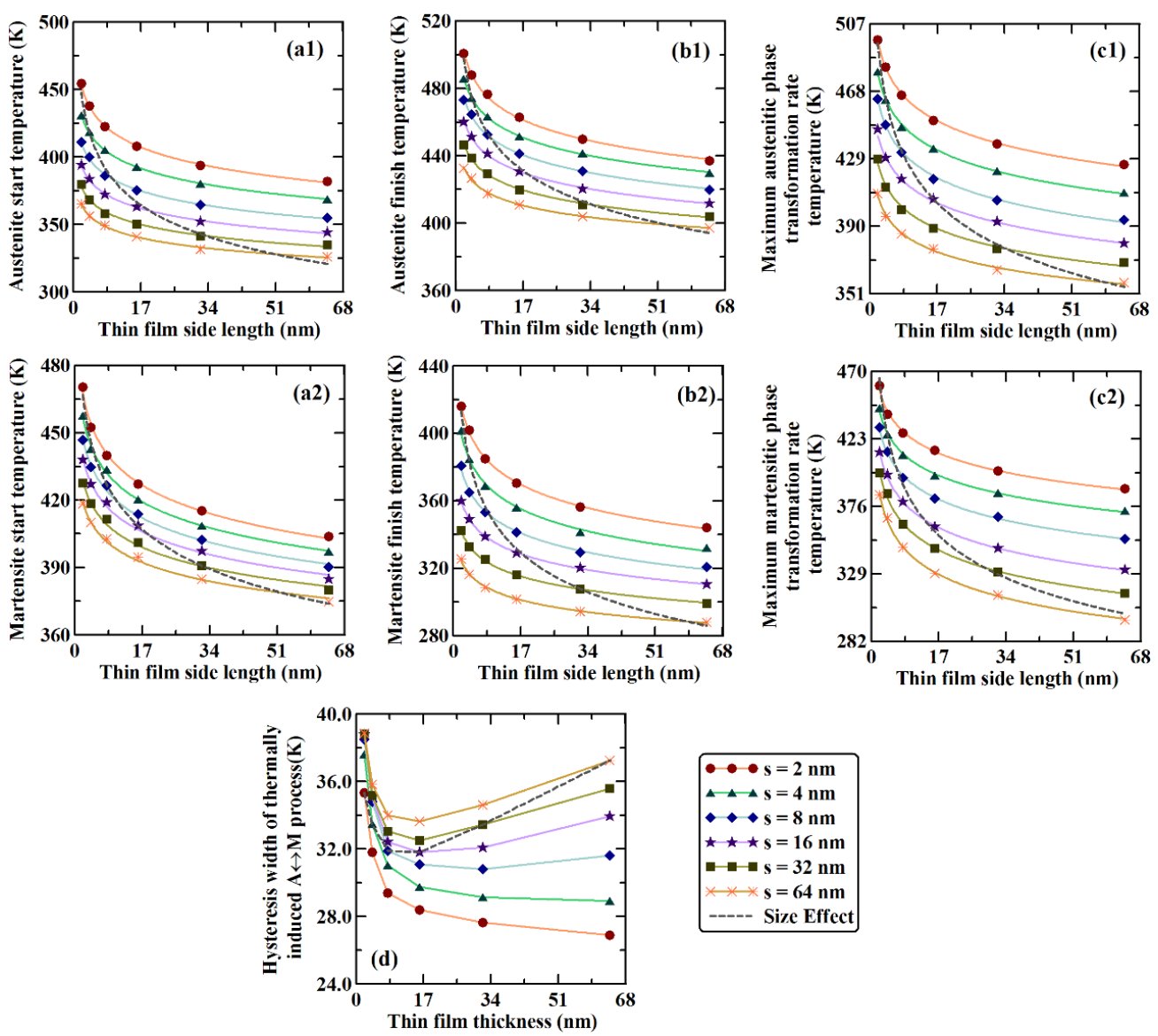

Figure-3: Effect of shape and size (i.e. both thickness and side length) on phase transformation temperatures and on thermal hysteresis width (a1) austenite start $\left(A_{s}\right)$, (b1) austenite finish $\left(A_{f}\right)$ and (c1) maximum austenitic phase transformation rate temperature $\left(T_{m A}\right)$; (a2) martensite start $\left(M_{s}\right)$, (b2) martensite finish $\left(M_{f}\right)$ and (c2) maximum martensitic phase transformation rate temperature $\left(T_{m M}\right)$; (d) width of thermal cycle between austenitic and martensitic phase transformation process.

Therefore, it is important to note that, in the present study, the reported size effects are due to the presence of free surfaces, which act as defects and trigger phase transformation. With decreasing simulation cell size, the surface area per unit volume increases, and this creates the dependency of the phase transformation process (transformation temperatures and phase fraction) on size. Mutter and Nielaba [11] and Zhang et al [13] demonstrate the phase transformation process for single crystal nano-scale NiTi simulation cells, by employing an EAM potential similar to the one used in this study. Importantly, in the Zhang et al [13], it is found that below size (diameter) of $1.15 \mathrm{~nm}$, no phase transformation occurs. In this study, the minimum size or side length of any simulation cell is adopted as $2.00 \mathrm{~nm}$, which is above $1.15 \mathrm{~nm}$. Finally, in the previous studies mentioned above, experiments and/or simulations are performed at constant temperature rates, which can play an important role in the phase transformation process, whereas in the present study quasi-static simulations (equilibrations) are performed at constant temperature. Thus, as potential future work, it will be very interesting to verify the model by employing experimental or simulation data for polycrystalline NiTi samples and obtain the model parameters.

\subsection{Length scale effects on phase fraction and internal strain evolution in single crystals}


The evolution of martensite phase faction and the development of internal strain in B19 $\square$ due to the evolving martensite phase are the two most important quantities that govern the NiTi material model. Thus, it is important to accurately study the evolution of these two internal variables and transfer all relevant information to the continuum scales via multiscale coupling.
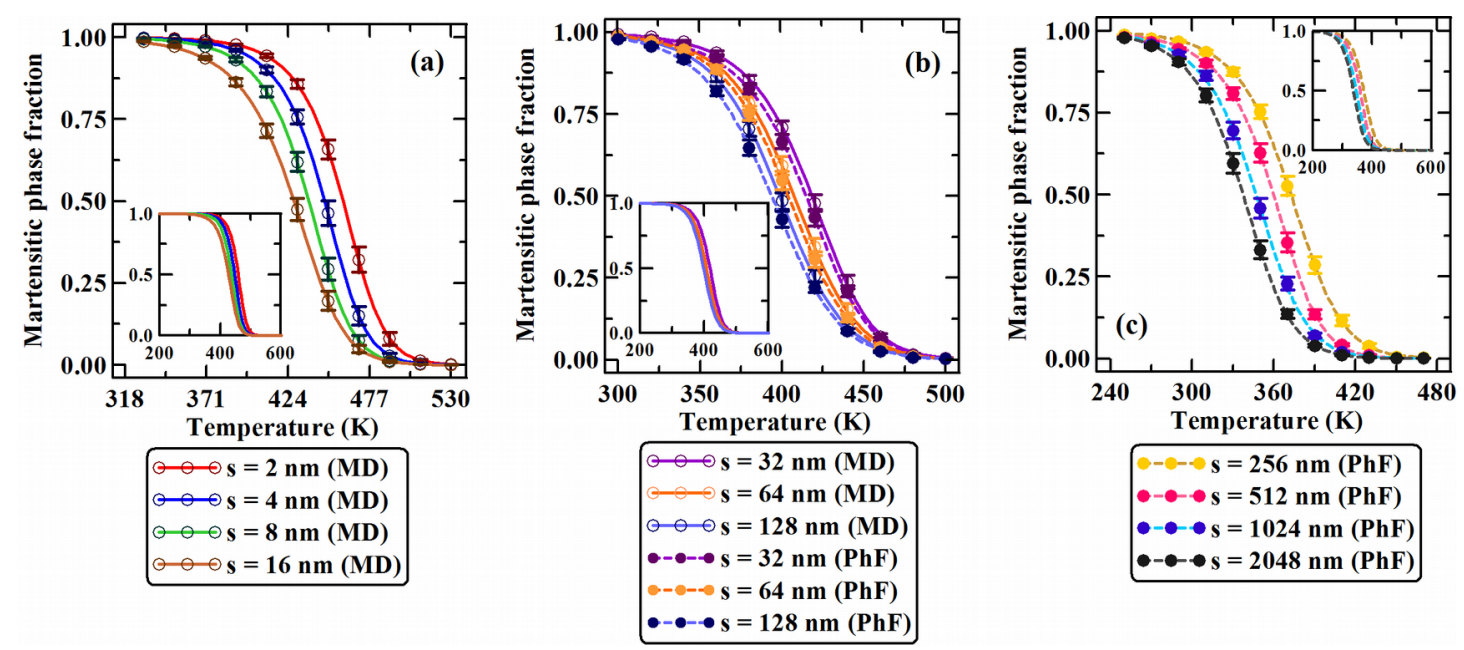

Figure-4: Evolution of martensite phase fraction with respect to temperature as a function of simulation cell size (a) within MD simulation domain, (b) within MD and PhF overlap simulation domain and (c) within PhF simulation domain.

Figure-4 shows the length scale effects at the mesoscale and their linkage to microscale i.e. atomistic simulation results. At the mesoscale, 2-D stochastic phase field $(\mathrm{PhF})$ simulation are performed and are linked to 3-D atomistic (MD) simulations. For all MD simulations, the thickness $t=2 \mathrm{~nm}$, and a range of side lengths, $s=2 i 128 \mathrm{~nm}$, is considered, with free boundary conditions in all directions. At the mesoscale level, the range of simulation cell sizes is from $s=32 i 2048 \mathrm{~nm}$, with free boundary conditions in all directions ( $\mathrm{x}, \mathrm{y}$ and $\mathrm{z}$ ). Therefore, the side length cases of $s=32 i 128 \mathrm{~nm}$ correspond to the MD and PhF overlap domain, where the two simulation methods are cross checked, showing very good agreement with each other. The error bars in the Fig. 4 (a)-(c) are the results of uncertainty in the evolution of different phases (B2 and $\mathrm{B} 19 \square$ variants). The uncertainty is quantified by performing a total of 25 Monte Carlo (MC) simulations at each temperature and for each size. In MD simulations, depending on the temperature, random velocities (based on Gaussian distribution) are provided to each atom and thus at the end of each simulation, the atomic coordinates are not the same as those obtained from other simulations at the same temperature and same size. Similarly at the beginning of stochastic $\mathrm{PhF}$ simulation, depending on the temperature, a Langevin noise term is added at every material point $[7,8]$. This causes small but noticeable differences in the evolution of martensite phase fraction, which ultimately produces uncertainties in the development of internal strain. Therefore, to account for those uncertainties, MC simulations are performed.

Another important parameter is the internal strain in B19 that governs the stability of different phases (i.e. B2 and different variants of B19 $)$ ). Since strong length scale effects are present in the phase evolution kinetics of NiTi (at both atomic and meso scales), it can be expected that similar effects should also be present for the transformation induced internal strain. However, this is not examined in the present work. In the MD simulations, the lattice 
parameters (bond lengths $a, b$ and $c$, monoclinic bond angle $\beta$ ) of the B19 $\square$ unit cell phase are measured. Here, it is important to mention that, in our MD simulations, with the adopted crystal orientation of B2 (i.e. [110], [ 1 10] and [001]) only two B19 variants are observed, which are favorable due to the requirement of low twining energy with respect to B2, as also reported in other MD studies [4,8,13-15] employing a similar interatomic potential. It is found from our MD simulations that, for the twin B19 $\square$ unit cell, the twinning energy is $E_{B 19}=-5.057 \mathrm{eV}$ with the difference in energy (i.e. the excess energy in reference to the single phase of $\mathrm{B} 19 \square$ ) of $\Delta E=-0.035 \mathrm{eV}$, which shows very good agreement with the values reported in the Zhong et al [4] study. Similarly, the twin boundary energy (i.e. the excess energy per unit boundary area of twin, in reference to the single phase of B19 $\square$ ) is also estimated and found that its value is very small compared to the other B19 variants for the twining case, i.e. $\gamma_{t}=-0.009 \mathrm{~J} / \mathrm{m}^{2}$ [4]. Finally, all of this indicates the possible reason for evolution of those two B19 $\square$ variants, in our, as well as in the other MD studies. Then, the deformation gradient matrix $\left(F_{i}\right)$ for the martensitic (B2 to any variants of B19 $\square$ ) phase transformation is estimated based on the lattice parameters of the B19 $\square$ unit cell phase and of the pristine $B 2$ phase $\left(a_{0}\right)$. Since the lattice parameters of the $B 19 \square$ phase change with length scale $(s)$ and temperatures $(T), \quad F_{i}$ becomes a function of $s$ and $T$. More information about $F_{i}$ for $\mathrm{B} 2 \square \mathrm{B} 19 \square$ phase transformation is available in previous studies [4,25]. Next, to determine the transformation induced strain tensor of different variants of the B19 $\square$ phase, the Green-Lagrange strain tensor $\left(\varepsilon_{i}^{0}\right)$ for each of the variants is estimated as

$$
\varepsilon_{i}^{0}(s, T)=\frac{1}{2}\left[\left\{F_{i}(s, T)\right\}^{T}\left\{F_{i}(s, T)\right\}-I\right]
$$

Finally, knowing the fraction of B19 $\square$ variants in the MD simulation cell (determined from the monoclinic bond angle $\beta$ ), at every temperature and for every size, the internal strain tensor in the $\mathrm{B} 19 \square$ phase is estimated through

$$
\varepsilon^{i}=\sum_{i=1}^{2} \psi_{i} \cdot \varepsilon_{i}^{0}=\frac{1}{2} \sum_{i=1}^{2} \psi_{i} \cdot\left[\left\{F_{i}(s, T)\right\}^{T}\left\{F_{i}(s, T)\right\}-I\right]
$$

Now, the mean value of the internal strain tensor of the B19 $\square$ phase $\left\langle\varepsilon^{i}\right\rangle$ and associated uncertainty (standard deviation) are estimated from 25 Monte Carlo (MC) MD simulations. Length scale effects in the average internal strain in the B19 $\square$ phase is due to the differential fraction evolution among the different B19 $\square$ variants. More information about the internal strain is provided in supplementary material. In PhF simulations, at any material point the internal strain associated with any B19 $\square$ variant ( $\varepsilon_{i}^{0}$ for the $i^{\text {th }}$ field variable) [7,26,27] and its fraction ( $\psi_{i}$ for the $i^{\text {th }}$ field variable), is used to estimate the total internal strain in B19 phase, as expressed trough $\varepsilon^{i}=\sum_{i=1}^{12} \psi_{i} \varepsilon_{i}^{0} \quad$ (for the 12 variants). Here it is important to mention that, although during the simulation process the possibility of evolution of all 12 B19 variants is considered, only four B19 $\square$ variants (1, 2, 6 and 8) are observed. It is due to the consideration of 2-D simulation cell, as discussed in [8]. Interestingly, it is found here that for both MD and PhF simulations, the mean internal strain tensor $\left(\varepsilon^{i}\right)$ is orthorhombic in 
nature. The mean value $\left\langle\varepsilon^{i}\right\rangle=\left\{\left(\sum_{k=1}^{M} \sum_{l=1}^{N} \varepsilon^{i}\right) /(M * N)\right\} \quad$ and its associated uncertainty is measured based on the number of MC simulations (here $M \quad 25$ ) and number of material points ( $N$ ) in each simulation cell. Since the fractional evolution of B19 $\square$ variants depends on the temperature and size of the simulation cell, the evolution of the average internal strain in the B19 phase will be a function of temperature and simulation cell size. Next, from the mean internal strain tensors $\left\langle\varepsilon^{i}\right\rangle$ in the B19 $\square$ phase, strains in three orthonormal directions (i.e. $\left\langle\varepsilon_{11}^{i}\right\rangle,\left\langle\varepsilon_{22}^{i}\right\rangle$ and $\left\langle\varepsilon_{33}^{i}\right\rangle$ ) are shown in Figure-5. This provides the direct estimation of temperature and size dependent lattice strains that B2 experiences during the martensitic phase transformation process. A recent relevant study by Ahadi and Sun [23] provides similar measures of lattice strains (in terms of stretches) and demonstrates the effect of grain size and applied strain on the evolution of lattice strain in NiTi SMA. As shown in Figure-5, strong interplay between the average internal strain in B19 and the length scale of the simulation cell is present. With increasing length scale, a strong decay is observed in all components of the maximum value of the average internal strain in B19 $\square$ i.e. $\left\langle\varepsilon_{i i}^{i}\right\rangle_{\max } \propto 1 / f|s\rangle$. It is clear that during the martensitic transformation process, the $[110]$ and $[001]$ directions of B2 experience compressive strain, while the [110] of B2 experiences tensile strain (as shown in Fig. 5 (c)). Interestingly, for any length scale, the temperature dependency of the normalized $\left\langle\varepsilon_{33}^{i}(s, T)\right\rangle$ in B19 (normalized with respect to the maximum value i.e. $\left\langle\varepsilon_{33}^{i}(s)\right\rangle_{\max }$ ) follows the same Richard's equation as the one for the evolution kinetics of the martensite phase fraction, i.e.

$$
\xi(s, T)=\frac{\left\langle\varepsilon_{33}^{i}(s, T)\right\rangle}{\left\langle\varepsilon_{33}^{i}(s)\right\rangle_{\max }}=1-\left[1+\exp \left\{-g \cdot v\left(T-T_{m}\right)\right\}\right]^{(-1 / v)}
$$

The other components of internal strain tensor i.e. $\left\langle\varepsilon_{11}^{i}\right\rangle$ and $\left\langle\varepsilon_{22}^{i}\right\rangle$ does not follow a relation similar to Eq. 25. The relevant direct relation between the martensite phase fraction and the internal strain tensor component $\varepsilon_{33}^{i}$, implies that $\varepsilon_{33}^{i}$ is mainly responsible for the stabilization of the B19 $\square$ variants and B2. Using a fitting process, it is found that the different parameters of the evolution equation (Eq. 25) i.e. $g, \quad v$ and $T_{m}$ vary with the length and can be expressed as

$$
\begin{gathered}
g(s)=g_{\text {bulk }}\left(\frac{\delta_{g}}{s}\right)^{\beta_{g}} \\
u(s)=v_{\text {bukl }}\left(\frac{s}{\delta_{v}}\right)^{\beta_{o}} \\
\frac{T_{m}(s)}{T_{m, \text { bulk }}}=\gamma_{m}-\beta_{m} \ln \left(\frac{s}{\delta_{m}}\right)
\end{gathered}
$$

where $g_{b u l k}\left(i K^{-1}\right)$ and $v_{b u k l}$ (unitless) are the constants corresponding to the bulk, $T_{m \text {,bulk }}$ is the temperature corresponding to the maximum martensite transformation rate of the bulk, $\beta_{g}$ (unitless) and $\beta_{v}$ (unitless) are constant for the power law exponent, $\delta_{g}$ (in $\mathrm{nm}$ ), $\delta_{v} \quad$ (in $\mathrm{nm}$ ) and $\delta_{m}$ (in $\mathrm{nm}$ ) are constants representing the length above which 
the length scale effect on $g, v$ and $T_{m}$ is negligible, and $\gamma_{m}$ and $\beta_{m}$ represent the intercept and slop of the $\left(\frac{T_{m}(s)}{T_{m, \text { bulk }}}\right)$ vs $\ln \left(\frac{s}{\delta_{m}}\right)$ line.
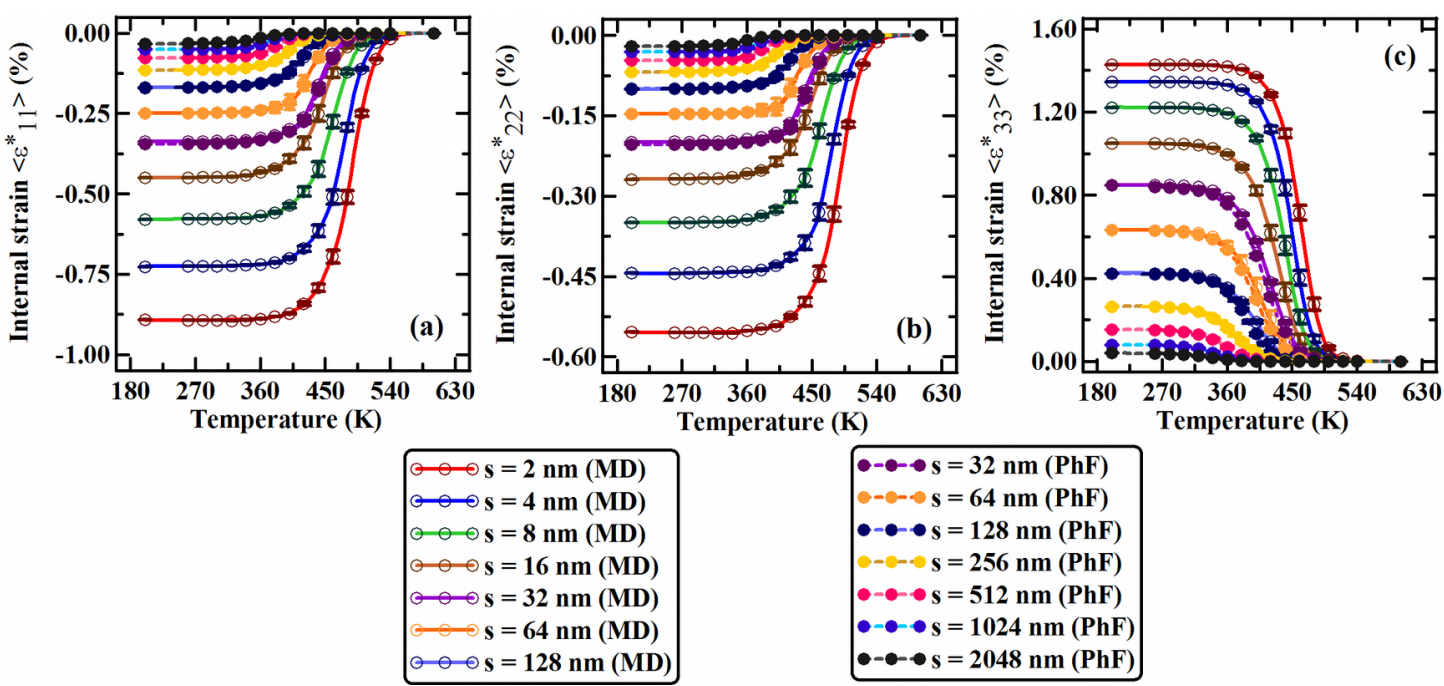

Figure-5: Evolution of average internal strains in $\mathrm{B} 19 \square$ with respect to temperature as a function of simulation cell length scale (a) $\left\langle\varepsilon_{11}^{i}\right\rangle$ in $[100]_{B 19^{\prime}}$ or $[110]_{B 2}$ direction, (b) $\left\langle\varepsilon_{22}^{i}\right\rangle$ in $[001]_{B 19^{\prime}}$ or $[001]_{B 2}$ direction and (b) $\left\langle\varepsilon_{33}^{i}\right\rangle$ in $[010]_{B 19^{\prime}}$ or $[110]_{B 2}$ direction; obtained from MD and $\mathrm{PhF}$ simulations.

Since the off diagonal terms of the mean internal strain tensor are negligible, the diagonal terms furnish the principal components of the internal strain tensor. Interestingly, it is found that while the diagonal terms are only present in the mean value of the transformation induced total internal strain tensor of the B19 $\square$ phase, the mean value of the total volumetric strain developed due to the martensitic phase transformation process (at any temperature and size) is almost zero, i.e. $\left\langle\varepsilon_{v}^{i}\right\rangle=\operatorname{tr}\left(\left\langle\varepsilon^{i}\right\rangle\right)=\left\langle\varepsilon_{11}^{i}\right\rangle+\left\langle\varepsilon_{22}^{i}\right\rangle+\left(\varepsilon_{33}^{i}\right\rangle \approx 0$.

Figure-6 shows the effects of length scale on the phase transformation temperatures (martensite start, maximum transformation rate, and finish in Fig. 6 (a)) and absolute maximum value of different elements of average internal strain tensor (i.e. $\mid\left\langle\varepsilon_{11}^{i} \|_{\max }\right.$, $\left|\left\langle\varepsilon_{22}^{i}\right\rangle\right|_{\max }$ and $\left|\left(\varepsilon_{33}^{i}\right\rangle\right|_{\max }$ ) in B19 (in Fig. 6 (b)). As can be observed from Fig. 6 (a), all transformation temperatures decrease with increasing length scale, following a linear trend with $\log$ of the length scale i.e. $\theta_{T}(s) \propto \ln (1 / s)$. Thus, each of the transformation temperatures can be described through an equation similar to Eq. (26.c), where the slope of the line is the same yet the intercepts are different. Similarly to the transformation temperatures, the absolute maximum value of each of the components of the internal strain tensor in $\mathrm{B} 19 \square$ phase decreases with length scale and can be expressed as

$$
\mid\left\langle\left.\varepsilon_{i i}^{i}(s)\right|_{\text {max }}=\gamma_{i i} \exp \left\{-\beta_{i i} \ln \left(\frac{s}{\delta_{i i}}\right)^{2}\right\} \text { for } i=1,2 \wedge 3\right.
$$

i.e. asymptotically converging to zero as size increases. Here, $\quad \gamma_{i i}$ (in \%), $\quad \beta_{i i}$ (unitless) and $\delta_{i i}$ (in nm) are constants. 

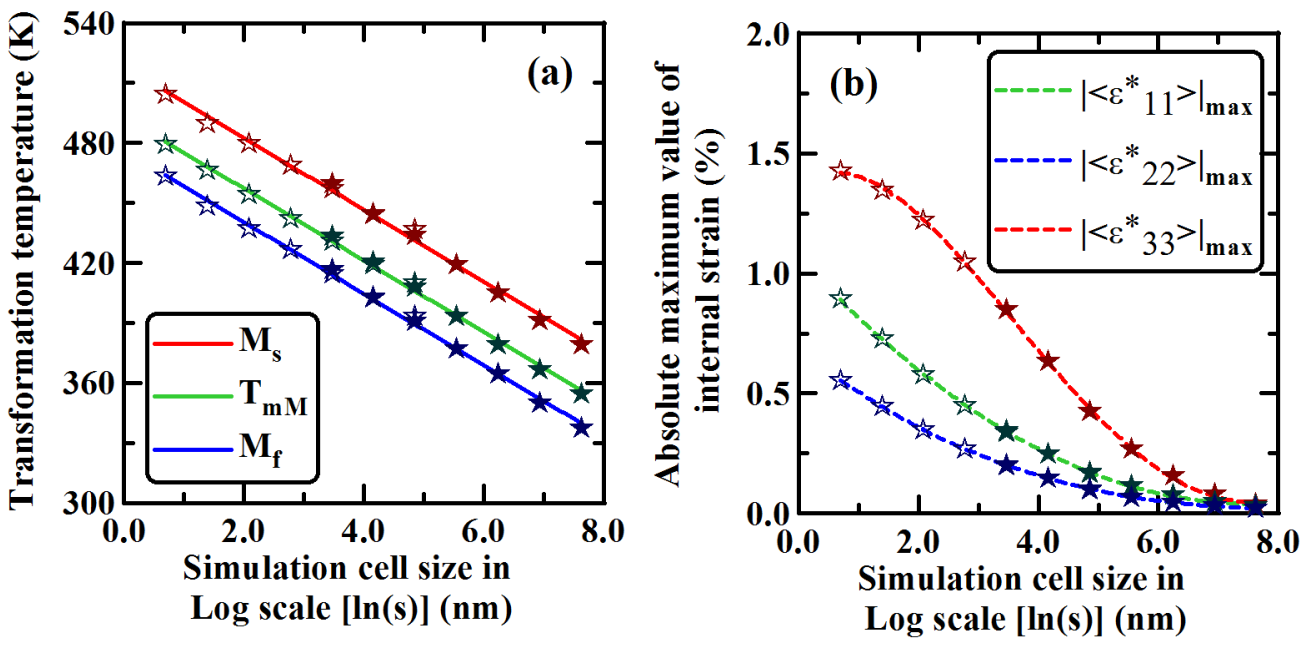

Figure-6: Variation of the (a) martensite start $\left(M_{s}\right)$, maximum martensitic transformation rate $\left(T_{m M}\right)$, martensite finish $\left(M_{f}\right)$ temperature and (b) absolute maximum value of different components of the average internal strain in B19 $\left(\right.$ i.e. $\quad\left|\left\langle\varepsilon_{11}^{i}\right\rangle\right|_{\max }, \quad\left|\left\langle\varepsilon_{22}^{i}\right\rangle\right|_{\max }$ and $\left|\left\langle\varepsilon_{33}^{i}\right\rangle\right|_{\max }$ ) due to thermally induced martensitic phase transformation with respect to the simulation length scale. Empty and filled stars correspond to results from $\mathrm{MD}$ and $\mathrm{PhF}$ simulations, respectively, while the regression lines (solid or dotted lines) result from a fitting process.

\subsection{Multiscale coupling of length scale effects}

This section describes the multiscale/ multiphysics coupling results for the thermally induced phase transformation process. Multiscale coupling via the CWM methodology is performed on the martensite phase fraction (MPF) evolution. Here, the statistical information of length scale effects on the MPF evolution kinetics (obtained via MD and PhF simulations), is transferred/ propagated from micro and meso scales to the macro scale. Furthermore, the CWM results are compared with continuum level experimental data and with macroscopic constitutive relations for NiTi SMA. Essentially, the continuum level experimental data/ evolution law predicts an exponential dependency of martensite phase fraction evolution on temperature, while the macroscopic constitutive relations are linear. Therefore, in this section, the experimental data is referred to as the 'exponential' model and MPF obtained from macroscopic constitutive relations is referred as the 'linear' model.

Figure-7 demonstrates the ability of the CWM method to transfer the trend and uncertainties of martensite phase fraction to the continuum level. In this context, it is important to noted that, the experimental sample is the polycrystalline with mostly contain [110] B2 crystals, i.e. similar like the CWM simulations. Thus, it can be expected that, in the CWM method, the effect of the grain boundary energy and internal strain, on the martensite phase fraction evolution is captured sufficiently, as is the case for the experiments. Fig. 7 (a) shows the evolution of martensite phase fraction as a function of temperature predicted by the CWM method, experimental data, described above in section 3.1, and from the continuum level thermo-mechanical constitutive relation (mentioned in section 3.2). It can be observed that the evolution of martensite phase fraction predicted by the CWM method matches very well the experimental data/ exponential model i.e. the blue curve [8,42]. Also, the CWM method transfers the statistics of length scale effects, as shown in Fig. 7 (a), in terms of the 
uncertainty bars on the CWM curve. However, the continuum level linear material model shows significant deviation from the experimental and/ or CWM results. To demonstrate the deviation, the differences between values from experimental data and from CWM and continuum material model are shown in Fig. 7 (b). The difference between experimental data/ exponential model and CWM predicted values is small or even negligible when uncertainties are considered, whereas this difference becomes very large for the case of experimental data and linear model obtained from continuum level thermo-mechanical constitutive relations.
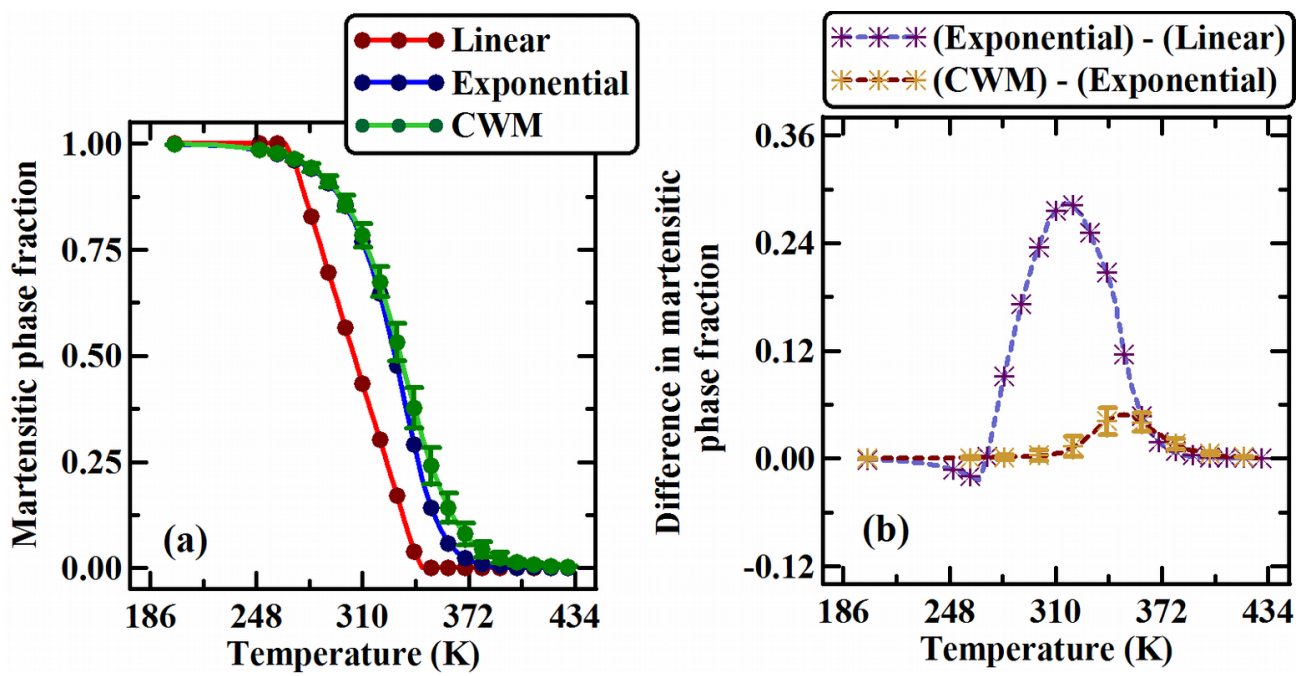

Figure-7 Variation of the (a) martensite phase fraction and (b) difference in martensite phase fraction with respect to the temperature, from different sub methods and material model. In Fig. 7 (a), the blue curve (exponential) represents the experimental data [8,42] and the red curve (linear) the one from macroscopic constitutive relations.

\subsection{Multiscale modeling and length scale and grain size effects in NiTi polycrystals}

The interplay of cell/specimen size and grain size on the thermo-mechanical properties such as phase transformation process in SMAs has received little attention despite its importance [16], perhaps because of the difficulties in addressing it. In some recent studies [1,21-23], attempts are made to model or experimentally demonstrate the effect of grain size on stress induced martensitic phase transformation (i.e. for superelasticity) in NiTi SMA. However, the interaction between sample size and grain size and their coupled effects on the martensitic phase transformation process was addressed in those studies. Therefore, differing from the previous studies [1,21-23], in this study, some attempts are made to shed the light on the effect of such interplay. Here, 2-D phase field (PhF) simulations are used to address the interplay of simulation cell size and grain size on the thermally induced MPF evolution, while for the multiscale modeling, simulation results (of different size single crystal) from both MD and PhF are adopted, as discussed subsequently.

In order to perform phase field simulation of polycrystalline $\mathrm{NiTi}$, simulation cells with the polycrystalline grain boundaries or interfaces are generated via Poisson-Voronoi tessellations, employing the MATLAB toolbox. To introduce the effect of grain boundary a gradient energy term is added, as mentioned in the Ahluwalia et al study [16]. This gradient energy term is expressed as the function of interfacial energy $\left(\gamma=0.9482 \mathrm{~J} / \mathrm{m}^{2}\right)$, width of the grain boundary $(\Delta)$ and field variable $\left(\eta_{i}\right)$. The value of the interfacial energy term $(\gamma)$ for all different type of interfaces (such as interface between all B2 and B19 $\square$ crystals, as well as 
B2-B19 interface) is considered the same, as assumed in other studies [7,16]. However, the effect of different B2 crystal orientation on the martensite phase fraction evolution is incorporated in the phase field model through the elastic strain energy term $[7,8,16]$. This elastic strain energy depends on the transformation strain tensors for B2 to B19 variants $\left(\varepsilon_{i}^{0}\right)$, which again depends on the crystal orientation (with respect to [100] B2 crystal) $[7,8,16]$. Therefore, before using those transformation strain tensors, they are rotated through the rotation matrix $R$; i.e. $\varepsilon_{i}^{0}$ is converted into $R \varepsilon_{i}^{0} R^{T} \quad[7,8,16]$. Here, this rotation matrix depends on the crystal orientation of a particular B2 grain, with respect to the [100] B2 crystal. Thus, based on the aforementioned process, the effects of interface/ grain boundary and crystal orientation are incorporated in the phase field model.

An indirect method (based on the multiscale approach) is used to mimic the results of polycrystalline NiTi SMA. For that, the wavelet coefficients of MPF from different size single crystal simulations (with the crystal orientation of [100], [110] and [111]) are used in a very unique way. Here, to construct the coefficients of the compound wavelet matrix (CWM) of the polycrystalline NiTi, wavelet coefficients of different single crystal NiTi are fused from different length scales, i.e. from different size samples. In case of single crystals (with orientations [100], [110] and [111]), depending on the size of the cells, simulations are performed employing MD and $\mathrm{PhF}$, as mentioned in section 2. It is important to mention that, before mixing the wavelet coefficients (obtained from different size single crystals of NiTi), they are multiplied by appropriate weight factors. These weight factors are adopted based on the fraction of the relevant crystal present in the simulation cell. Adopting these weight factors is important, since they act as a step up or step down operator and thus amplify or reduce the value of the wavelet coefficients of single crystal NiTi, such that similar (to a close approximation) wavelet coefficients of polycrystalline NiTi are obtained. Since, in this indirect method the CWM is constructed, it is referred to as the 'Hybrid CWM' and this term is used in subsequently.

To demonstrate the coupled effect of grain size and length scale on MPF evolution and its associated multiscale modeling, a simulation cell of size $s=512 \mathrm{~nm}$ is adopted and simulations are performed at four different temperatures $(T=300,313,325$ and $350 \mathrm{~K})$ with different grain sizes. Figure 8 shows the effect of grain size on the thermally induced MPF evolution, for simulation cell size $s=512 \mathrm{~nm}$. The average values of the grain size and corresponding MPF are directly plotted in Figure 8, while their corresponding uncertainties are represented through the error bars. In order to directly compare the single crystal and polycrystal simulations, MPF values (mean and standard deviation) are provided for the four different temperatures. Fig. 8 (a) shows the coupled effects of grain size and simulation cell size, on the thermally induced MPF evolution, at $\mathrm{T}=313 \mathrm{~K}$. Also, Fig. 8 (a) demonstrates the efficiency of the hybrid CWM method in mimicking the MPF evolution of polycrystalline NiTi SMA as a function of grain size, for $\mathrm{T}=313 \mathrm{~K}$. It is seen that the MPF obtained from the direct polycrystal 2-D PhF simulations and the hybrid CWM method are in very good agreement with each other. Further, those results are compared with a reported computational study [16] and an experimental study [42]. Results from the computational study [16] agree very well with our direct polycrystalline NiTi simulation, as well as with the hybrid CWM predictions. However, the experimental value stays a little off from all other cases. Such deviation can be attributed to size effects for the NiTi samples/ cells and due to the constant 
temperature simulation. The experiments [42] were conducted at the continuum level, with a fixed temperature rate $\left(20^{\circ} \mathrm{C} / \mathrm{min}\right.$ cooling and heating rate).

Next, to conform and emphasize the efficiency and prediction capability of the hybrid CWM method, results are generated at different temperatures $(\mathrm{T}=300,325$ and $350 \mathrm{~K})$ and compared to the direct simulation results of polycrystalline NiTi SMA. As shown in Figs. 8 (b)-(d), the hybrid CWM results agree very well with the direct polycrystalline simulation results. Further, the deviation of the experimental MPF values from the simulation ones (from both the direct simulation and hybrid CWM) decreases with increasing temperature. For all examined temperatures, when the results from polycrystalline NiTi are compared to the results from single crystal NiTi, a significant difference in the MPF value is observed. Such noticeable difference in MPF value arises from the presence of grain boundary. Further, the simulations confirm that with the reduction in grain size, the evolution of the MPF decreases drastically and thus below a critical grain size martensitic phase transformation will not be observed in case of the polycrystalline NiTi SMA, as reported in many other studies $[1,10,17-19,21-23]$.
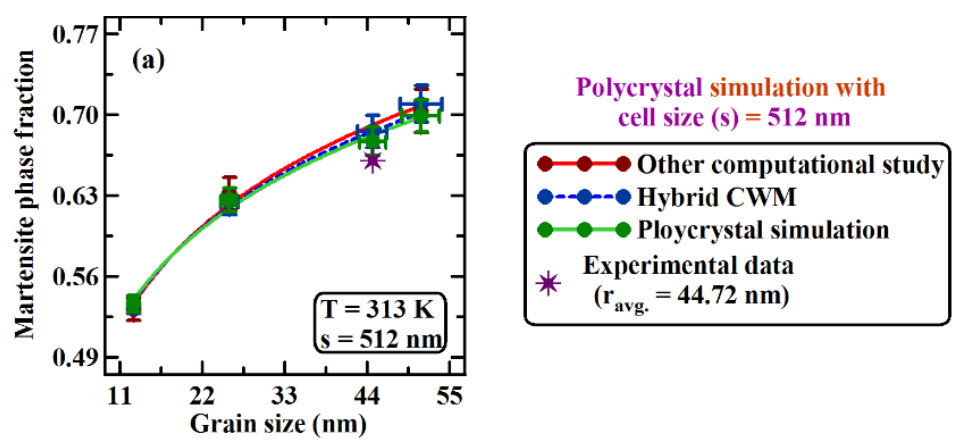

Single crystal simulation results with cell size $(\mathrm{s})=\mathbf{5 1 2} \mathrm{nm}$

Martensite phase fraction $(\xi)$ at different temperature $(\mathrm{T})$ $\mathrm{T}=300 \mathrm{~K} ; \xi=0.968 \pm 0.024$ $\mathrm{T}=313 \mathrm{~K} ; \xi=0.893 \pm 0.038$ $\mathrm{T}=325 \mathrm{~K} ; \xi=0.824 \pm 0.045$ $\mathrm{T}=350 \mathrm{~K} ; \xi=0.608 \pm 0.061$
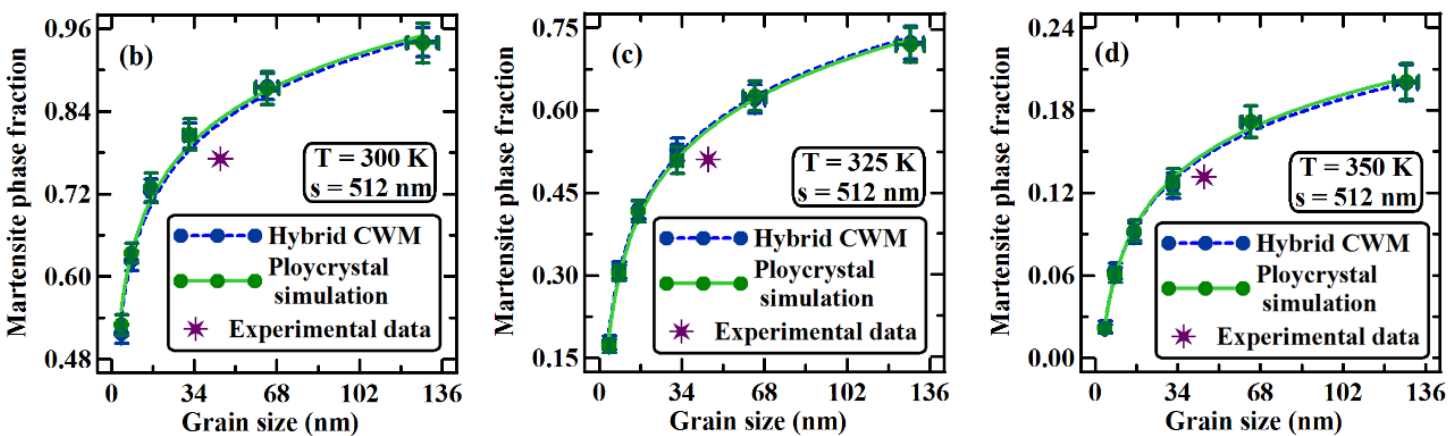

Figure-8 Variation of martensite phase fraction as a function of grain size for polycrystalline simulation cell of size $s=512 \mathrm{~nm}$, at temperature (a) $T=313 \mathrm{~K}$, (b) $T=300 \mathrm{~K}$, (c) $T=325 K$ and (d) $T=350 K$. Other study result (computational study for the polycrystalline NiTi) and experimental data are from the Refs. [16] and [42], respectively. Deviation between experiments and simulations is attributed to size effects as discussed in the text.

The above results show the coupled effect of grain size and simulation cell size on thermally induced MPF evolution, but do not clearly show the interplay between grain size and cell size and their effect on the MPF evolution. Figure 9 shows such interplay for three different temperatures ( $\mathrm{T}=300,325$ and $350 \mathrm{~K}$ ), the interplay becoming prominent for smaller grain sizes. To clearly investigate the interplay, direct polycrystalline $\mathrm{PhF}$ simulations are performed. For each temperature (and mainly for $\mathrm{T}=300$ and $325 \mathrm{~K}$ ), with the increase in simulation cell size (i.e. removal of length scale effects), the grain size effect becomes more 
pronounced, causing a drastic decrease in MPF evolution with decrease in grain size. This can be explained as follows. For larger simulation cell with smaller grain size, the effective amount (length) of grain boundary is high, which increases the total grain boundary energy. High grain boundary energy provides a driving force opposing the martensitic phase transformation process and thereby prevents the nucleation and growth of martensite (different variants of B19 7 ) from austenite (B2). Interestingly, it can be observed that with the increase in simulation cell size, as the length scale effect is removed, polycrystalline NiTi simulation results start agreeing very well with experimental data. To further demonstrate the effect of grain size in polycrystalline NiTi, results of single crystal NiTi simulations are also shown in Figure 9. Finally, the relation between normalized MPF and normalized grain size can be express as

$$
\frac{\xi_{p}\left(s, s_{g}, T\right)}{\xi(s, T)}=\gamma_{p}+\beta_{p} \ln \left(\frac{s_{g}}{s}\right)
$$

where, $\xi_{p}\left(s, s_{g}, T\right)$ and $\xi(s, T)$ are the MPFs corresponding to polycrystalline and single crystal NiTi SMA, respectively, $s_{g}$ and $s$ are the grain size and simulation cell size, respectively, $\gamma_{p}$ is a unitless constant which represent the normalized MPF value for a sample with very large grain size, and $\beta_{p}$ is a constant representing the normalized MPF and normalized grain size (in log scale) curve.
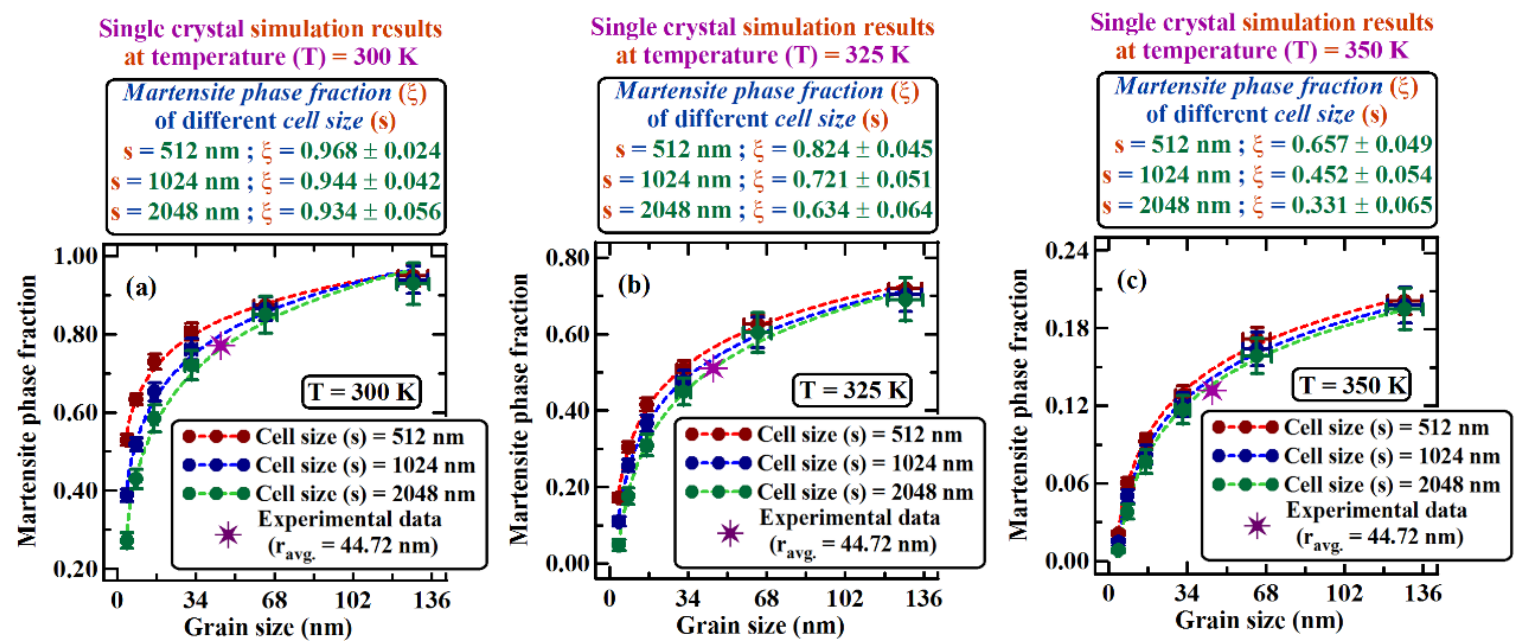

Figure-9 Variation of martensite phase fraction as a function of grain size for polycrystalline simulation cell of size $s=512,1024 \wedge 2048 \mathrm{~nm}$, shows the interplay of grain size and the simulation cell size on martensite phase fraction evolution, at temperature (a) $T=300 \mathrm{~K}$, (b) $T=325 \mathrm{~K}$ and (c) $T=350 \mathrm{~K}$.

\subsection{Stress-strain response}

This section describes the application of multiscale coupling to macroscopic continuum scale constitutive relations. In order to improve the continuum thermo-mechanical material model of NiTi SMA described previously, the temperature dependent martensite phase fraction and its spatial uncertainties (obtained from multiscale coupling) are transferred into the continuum constitutive model. In the present version of the continuum model it is assumed that the martensite phase fraction varies linearly with temperature. 
Figure-10 shows the stress strain hysteretic loops and the associated energies obtained from different methods and from experimental data. The linear evolution law for the thermally induced martensite phase fraction shows noticeable deviation from the experimental stressstrain data, while the exponential evolution law (expressed in Eq. 20) and the CWM provided thermally induced martensite phase fraction show very good agreement with the experimental data. The same holds for energy dissipation capacity of NiTi SMA (Fig. 10 (b) for single cycle and Fig. 10 (c) for multiple cycles). Thus, the improvement in the material modeling of NiTi SMA is rather significant, at least for those cases where NiTi SMA is used as an energy dissipative medium, such as in NiTi SMA based vibration dampers or actuators.
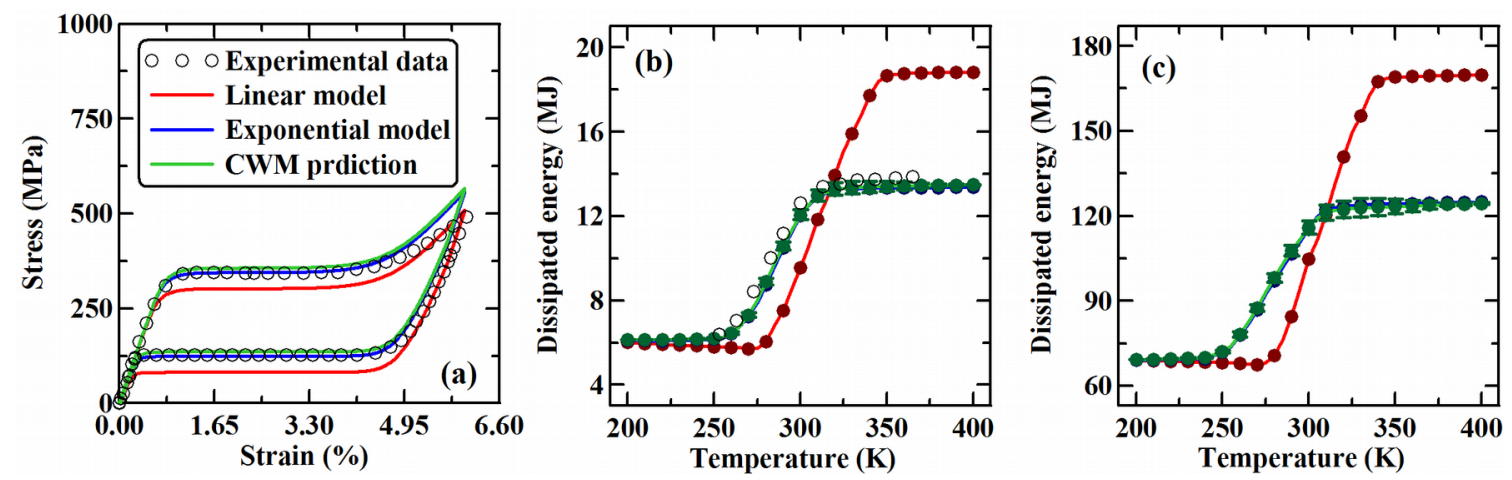

Figure-10: (a) Stress strain hysteresis loop of NiTi at $\mathrm{T}=300 \mathrm{~K}$ obtained by employing different methods and experimental data. Comparison of hysteresis energy dissipation capacity at various temperatures (b) in single cycle and (c) in multiple cycles.

The improvement in the continuum material model is demonstrated for other temperature cases in Figure-11. It can be observed that within the transformation temperature zone, the stress-strain hysteresis loops obtained from the linear evolution law deviates from that obtained from CWM and from exponential evolution law. However, outside the transformation temperature regime, such differences in the stress-strain hysteresis loops diminish. Here at $\mathrm{T}=200 \mathrm{~K}$, the martensite phase fraction obtained from different models is the same, thus in Fig. 11 (a) stress-strain hysteresis loop obtained from different models are indistinguishable. 

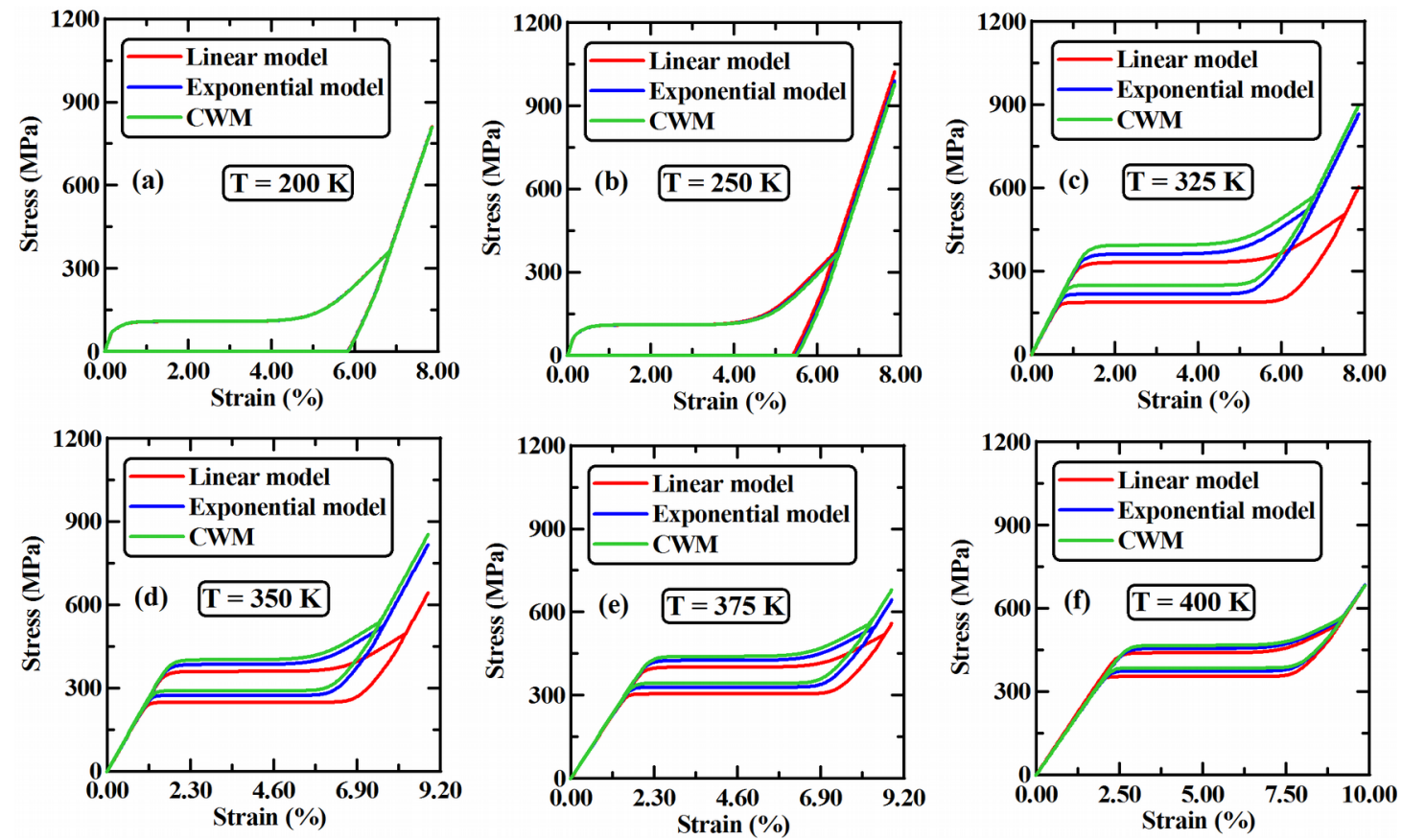

Figure-11: Constitutive behavior of the NiTi SMA at different temperatures obtained from different methods.

The uncertainties associated with thermally induced martensite phase fraction obtained from the multiscale coupling are used to demonstrate the hysteretic stress-strain response of NiTi SMA at different temperatures. In Figure-12, at each temperature, five curves are plotted, i.e. at mean phase fraction value and at mean with two different levels of uncertainties $(\mu, \mu \pm \sigma, \mu \pm 2 \sigma)$. It can be observed that within the transformation temperature regime, uncertainty in the thermally induced martensite phase fraction has a pronounced effect on the stress-strain response. However, this effect of uncertainties diminishes outside the transformation temperature zone.
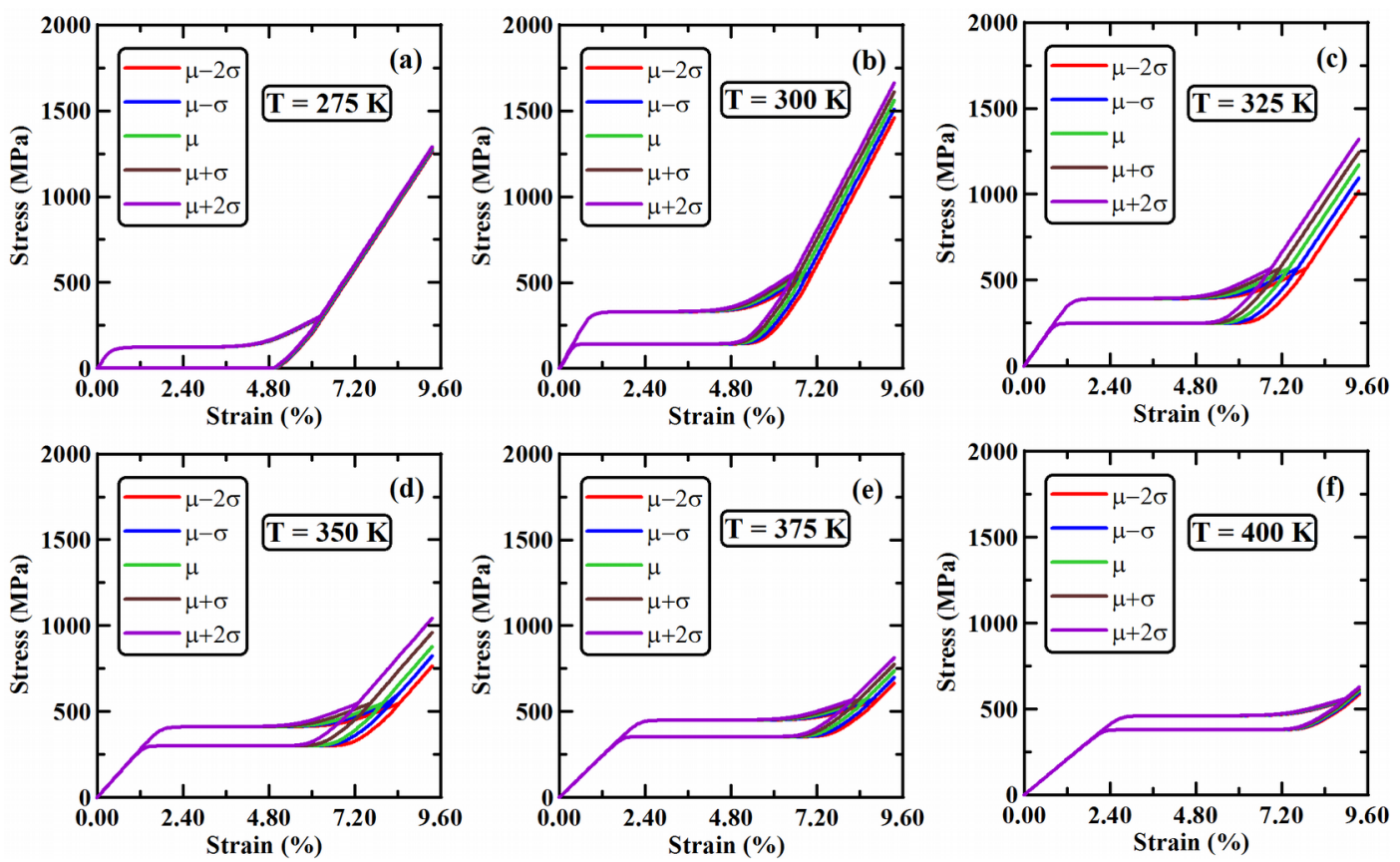
Figure-12: Effect of uncertainties in the thermally induced martensite phase fraction on the stress-strain response of NiTi SMA at different temperatures.

\section{Conclusions}

This study demonstrates the effects of length scale (i.e. shape and size effects) on thermally induced martensite phase fraction evolution kinetics in NiTi SMA and implements a relevant multiscale and multiphysics model. To study the evolution kinetics of B2 and of B19 variants, different methods, molecular dynamics (MD) simulations for fine scale, phase field $(\mathrm{PhF})$ simulations for meso scale, and experimental data for continuum level, are used. The lattice Boltzmann method (LBM) is employed for simulating the heat diffusion process in NiTi thin samples and multiscale coupling is performed through the compound wavelet matrix (CWM) method. Results of this study show strong length scale effects, which are linked to the presence of free surfaces. Change of size and shape of NiTi thin samples causes substantial changes in the transformation temperatures, as well as in the width of thermal cycles. With increasing side length and thickness of the thin sample, the transformation temperatures decrease. The temperature dependent evolution of martensite phase fraction follows the phenomenological Richard's equation. Strong length scale effects are also noticed for the evolution kinetics of the average internal strain in the B19 $\square$ phase, and its maximum value decreases exponentially with increasing simulation cell size. Interestingly, it is found that, the same Richard's equation can be used to express the temperature dependency of the normalized average internal strain in the $\mathrm{B} 19 \square$ phase. Through multiscale coupling, the length scale effects on thermally induced martensite phase fraction evolution and its associated uncertainties are propagated into the continuum level and used to improve existing constitutive models for NiTi SMA. The efficiency and robustness of the multiscale modeling method is further demonstrated for polycrystalline NiTi samples at different temperatures, and shows the coupled effects of grain size, simulation cell size, and temperature on the martensite phase fraction evolution kinetics. The interplay between grain size and length scale on the thermally induced MPF evolution shows that for large simulation cell size (where the length scale effect is negligible) and small grain size, the martensitic phase transformation process arrests. The effect becomes more pronounced for large size simulation cells, where, with decreasing grain size, the MPF decreases drastically. Employing the multiscale modeling results, the continuum level thermo-mechanical constitutive relation is modified appropriately. The modified material model significantly improves the predicted stress-strain response and energy dissipation capacity of NiTi SMA and shows good agreement with experimental data. Furthermore, the uncertainties in martensite phase fraction evolution show noticeable effects in the macroscopic stress-strain response. It can be concluded that improvement in material model and consideration of such uncertainties are important, at least for designing NiTi SMA base devices such as dampers and actuators. In future work, it will be interesting to study the interplay of grain size in polycrystalline NiTi SMA and cell size in the evolution kinetics (nucleation and growth of different phases) and their effect on phase transformation temperatures.

\section{Acknowledgement}

The reported exploratory work became possible through the support of SG by the University of Arizona. Also the authors would like to acknowledge Prof. Ting Zhu (from Woodruff 
school of mechanical engineering at Georgia Institute of Technology) for providing the interatomic potential parameters.

\section{References}

[1] Sun Q P and He Y J 2008 A multiscale continuum model of the grain-size dependence of the stress hysteresis in shape memory alloy polycrystals International Journal of Solids and Structures 45 3868-96

[2] Yang L and Dayal K 2010 Formulation of phase-field energies for microstructure in complex crystal structures Applied Physics Letters 96 081916-9

[3] Roubíček T 2011 Approximation in multiscale modelling of microstructure evolution in shape-memory alloys Continuum Mechanics and Thermodynamics 23 491-507

[4] Zhong Y, Gall K and Zhu T 2011 Atomistic study of nanotwins in NiTi shape memory alloys Journal of Applied Physics 110033532

[5] Salman O U, Finel A, Delville R and Schryvers D 2012 The role of phase compatibility in martensite Journal of Applied Physics 111103517

[6] Benafan O, Padula S A, Noebe R D, Sisneros T A and Vaidyanathan R 2012 Role of B19' martensite deformation in stabilizing two-way shape memory behavior in NiTi Journal of Applied Physics 112093510 (11pp)

[7] Zhong Y and Zhu T 2014 Phase-field modeling of martensitic microstructure in NiTi shape memory alloys Acta Materialia 75 337-47

[8] Gur S and Frantziskonis G N 2016 Linking simulations and experiments for the multiscale tracking of thermally induced martensitic phase transformation in NiTi SMA transformation in NiTi SMA Modelling and Simulation in Materials Science and Engineering Modelling 24075006 (26pp)

[9] Fu Y Q, Zhang S, Wu M J, Huang W M, Du H J, Luo J K, Flewitt a. J and Milne W I 2006 On the lower thickness boundary of sputtered TiNi films for shape memory application Thin Solid Films $\mathbf{5 1 5}$ 80-6

[10] Waitz T, Antretter T, Fischer F D and Karnthaler H P 2008 Size effects on martensitic phase transformations in nanocrystalline NiTi shape memory alloys Materials Science and Technology 24 934-40

[11] Mutter D and Nielaba P 2011 Simulation of the thermally induced austenitic phase transition in NiTi nanoparticles The European Physical Journal B 84 109-13

[12] Mutter D and Nielaba P 2013 Simulation of the shape memory effect in a NiTi nano model system Journal of Alloys and Compounds 577 S83-7

[13] Zhang Z, Ding X, Deng J, Cui J, Sun J, Suzuki T, Otsuka K and Ren X 2013 Surface Effects on Structural Phase Transformations in Nanosized Shape Memory Alloys The Journal of Physical Chemistry C 117 7895-901 
[14] Mirzaeifar R, Gall K, Zhu T, Yavari A and Desroches R 2014 Structural transformations in NiTi shape memory alloy nanowires Journal of Applied Physics 115 194307 (8pp)

[15] Gur S and Frantziskonis G N 2016 Atomistic study on size effects in thermally induced martensitic phase transformation of NiTi Smart Materials Research 20167512642 (12pp)

[16] Ahluwalia R, Quek S S and Wu D T 2015 Simulation of grain size effects in nanocrystalline shape memory alloys Journal of Applied Physics 117244305 (13pp)

[17] Waitz T, Pranger W, Antretter T, Fischer F D and Karnthaler H P 2008 Competing accommodation mechanisms of the martensite in nanocrystalline NiTi shape memory alloys Materials Science and Engineering: A 481 479-83

[18] Waitz T, Spisak D, Hafner J and Karnthaler H P 2005 Size-dependent martensitic transformation path causing atomic-scale twinning of nanocrystalline NiTi shape memory alloys Europhysics Letters (EPL) 71 98-103

[19] Waitz T, Antretter T, Fischer F D, Simha N K and Karnthaler H P 2007 Size effects on the martensitic phase transformation of NiTi nanograins Journal of the Mechanics and Physics of Solids 55 419-44

[20] Wu C-D, Sung P-H and Fang T-H 2013 Study of deformation and shape recovery of NiTi nanowires under torsion Journal of Molecular Modeling 19 1883-90

[21] Ahadi A and Sun Q 2013 Stress hysteresis and temperature dependence of phase transition stress in nanostructured NiTi - Effects of grain size Applied Physics Letters 103021902 (5pp)

[22] Ahadi A and Sun Q 2014 Effects of grain size on the rate-dependent thermomechanical responses of nanostructured superelastic NiTi Acta Materialia 76 186-97

[23] Ahadi A and Sun Q 2015 Stress-induced nanoscale phase transition in superelastic NiTi by in situ X-ray diffraction Acta Materialia 90 272-81

[24] Gur S, Manga V R, Bringuier S, Muralidharan K and Frantziskonis G 2015 Anisotropy in the Transformation Dynamics of Austenite (B2) to Martensite (B19') Associated with Superelasticity in NiTi 2015 TMS Annual Meeting \& Exhibition (Orlando, FL, USA)

[25] Knowles K M and Smith D A 1981 The crystallography of the martensitic transformation in equiatomic nickel-titanium Acta Metallurgica 29 101-10

[26] Hane K F and Shield T W 1999 Microstructure in the cubic to monoclinic transition in titanium-nickel shape memory alloys Acta Materialia 47 2603-17

[27] James R D and Hane K F 2000 Martensitic transformations and shape-memory materials Acta Materialia 48 197-222 
[28] Dai H and Frantziskonis G 1994 Heterogeneity, spatial correlations, size effects and dissipated energy in brittle materials Mechanics of Materials 18 103-18

[29] Frantziskonis G N 2013 Multiscale unified prediction of size / scale and Hall-Petch effects in the mechanics of polycrystalline materials Journal of the Mechanical Behavior of Materials 22 67-71

[30] Vvedensky D D 2004 Multiscale modelling of nanostructures Journal of Physics: Condensed Matter 16 R1537-76

[31] Hariharan G and Kannan K 2014 Review of wavelet methods for the solution of reaction-diffusion problems in science and engineering Applied Mathematical Modelling 38 799-813

[32] Tadmor E, Phillips R and Ortiz M 1996 Mixed atomistic and continuum models of deformations in solids Journal of the ACM 7463 4529-34

[33] Kevrekidis I G, Gear C W, Hyman J M, Kevrekidis P G, Runborg O and Theodoropoulos C 2002 Equation-Free Multiscale Computation: enabling microscopic simulators to perform system-level tasks Communications in Math Sci 1 715-62

[34] Dollet A 2004 Multiscale modeling of CVD film growth-a review of recent works Surface and Coatings Technology 177-178 245-51

[35] Frantziskonis G and Deymier P A 2000 Wavelet methods for analysing and bridging simulations at complementary scales - the compound wavelet matrix and application to microstructure evolution Modelling and Simulation in Materials Science and Engineering 8 649-64

[36] Frantziskonis G 2002 Wavelet-based analysis of multiscale phenomena: Application to material porosity and identification of dominant scales Probabilistic Engineering Mechanics 17 349-57

[37] Mishra S K, Muralidharan K, Deymier P A and Frantziskonis G N 2008 Wavelet-Based Spatial Scaling of Coupled Reaction-Diffusion Fields International Journal for Multiscale Computational Engineering 6 281-97

[38] Frantziskonis G and Deymier P A 2003 Wavelet-based spatial and temporal multiscaling: Bridging the atomistic and continuum space and time scales Physical Review B 68024105 (8pp)

[39] Muralidharan K, Mishra S K, Frantziskonis G, Deymier P A, Nukala P, Simunovic S and Pannala S 2008 Dynamic compound wavelet matrix method for multiphysics and multiscale problems Physical Review E 77026714 (14pp)

[40] Frantziskonis G, Muralidharan K, Deymier P A, Simunovic S, Nukala P and Pannala S 2009 Time-parallel multiscale/multiphysics framework Journal of Computational Physics 228 8085-92 
[41] Gur S, Danielson T, Xiong Q, Hin C, Pannala S, Frantziskonis G, Savara A and Daw C S 2016 Wavelet-based surrogate time series for multiscale simulation of heterogeneous catalysis Chemical Engineering Science 144 165-75

[42] Zotov N, Marzynkevitsch V and Mittemeijer E J 2014 Evaluation of kinetic equations describing the martensite-austenite phase transformation in NiTi shape memory alloys Journal of Alloys and Compounds 616 385-93

[43] Lai W S and Liu B X 2000 Lattice stability of some Ni-Ti alloy phases versus their chemical composition and disordering Journal of Physics: Condensed Matter 12 L5360

[44] Plimpton S 1995 Fast Parallel Algorithms for Short-Range Molecular Dynamics Journal of Computational Physics 117 1-19

[45] Zhong Y, Gall K and Zhu T 2012 Atomistic characterization of pseudoelasticity and shape memory in NiTi nanopillars Acta Materialia 60 6301-11

[46] Gur S, Mishra S K and Frantziskonis G N 2015 Thermo-mechanical strain ratedependent behavior of shape memory alloys as vibration dampers and comparison to conventional dampers Journal of Intelligent Material Systems and Structures 27 125064

[47] He X, Chen S and Doolen G D 1998 A Novel Thermal Model for the Lattice Boltzmann Method in Incompressible Limit Journal of Computational Physics 146 282-300

[48] Peng Y, Shu C and Chew Y T 2003 Simplified thermal lattice Boltzmann model for incompressible thermal flows Physical Review E 68026701 (8pp)

[49] D’Orazio a., Succi S and Arrighetti C 2003 Lattice boltzmann simulation of open flows with heat transfer Physics of Fluids 15 2778-81

[50] Wang J, Wang M and Li Z 2007 A lattice Boltzmann algorithm for fluid-solid conjugate heat transfer International Journal of Thermal Sciences 46 228-34

[51] Mishra S K, Deymier P A, Muralidharan K, Frantziskonis G, Pannala S and Simunovic S 2010 Modeling the coupling of reaction kinetics and hydrodynamics in a collapsing cavity Ultrasonics Sonochemistry 17 258-65

[52] Frantziskonis G N 2011 Lattice Boltzmann method for multimode wave propagation in viscoelastic media and in elastic solids Physical Review E 83066703 (8pp)

[53] Mishra S K and De A 2013 Coupling of reaction and hydrodynamics around a reacting block modeled by lattice Boltzmann method (LBM) Computers \& Fluids 71 91-7

[54] Zanotti C, Giuliani P, Riva G, Tuissi a. and Chrysanthou A 2009 Thermal diffusivity of Ni-Ti SMAs Journal of Alloys and Compounds 473 231-7 
[55] Zanotti C, Giuliani P, Bassani P, Zhang Z and Chrysanthou A 2010 Comparison between the thermal properties of fully dense and porous NiTi SMAs Intermetallics 18 $14-21$

[56] Zanotti C, Giuliani P and Chrysanthou A 2012 Martensitic-Austenitic phase transformation of Ni-Ti SMAs: Thermal properties Intermetallics 24 106-14

[57] Fallis A . 1996 Kinetic model of thermoelastic martensite transformation in NiTi and NiMn based shape memory alloys MRS Symp. Proc 398 537-42

[58] Lai B-K, Kahn H, Phillips S M, Akase Z and Heuer a. H 2011 Quantitative phase transformation behavior in TiNi shape memory alloy thin films Journal of Materials Research 19 2822-33

[59] Frantziskonis G N and Gur S 2017 Computational simulations for the development of novel solid- state smart NiTi-Al thermal diodes Journal of Intelligent Material Systems and Structures DOI: 10.1177/1045389X16685440, 2017 (13 pp).

[60] Helm D and Haupt P 2003 Shape memory behaviour: modelling within continuum mechanics Int. J. Sol. Struct. 40 827-49 